\title{
A CONSTRUCTIVE APPROACH TO THE UNIVERSALITY CRITERION FOR SEMIGROUPS
}

\author{
David Walmsley
}

\author{
A Dissertation \\ Submitted to the Graduate College of Bowling Green \\ State University in partial fulfillment of \\ the requirements for the degree of

\section{DOCTOR OF PHILOSOPHY}

May 2017

Committee:

Kit Chan, Advisor

John Laird,

Graduate Faculty Representative

Juan Bes

Alexander Izzo 
Copyright (C)May 2017

David Walmsley

All rights reserved 


\section{ABSTRACT}

Kit Chan, Advisor

We prove a generalization of the well-known Universality Criterion in the setting of continuous homomorphisms acting on a separable, complete, metrizable topological semigroup by constructing a particular universal element. Consequently, we simplify the proof of a 1955 classical result of Heins on the existence of universal Blaschke products on the unit ball of $H^{\infty}(\mathbb{D})$, and the proofs of a number of other related results, by unifying them in the semigroup setting. Motivated by our generalization, we provide new applications such as the homomorphisms of conjugation on the unit ball of the operator algebra of $c_{0}$ and $\ell^{p}$, where $1 \leq p<\infty$, and also the homomorphisms of composition on the closed unit ball $\overline{\mathrm{Ball}} L^{\infty}[0,1]$ of $L^{\infty}[0,1]$ with the weak-star topology, as well as on the semigroup $L^{1}(\mathbb{R}) \cap \overline{\mathrm{Ball}} L^{\infty}(\mathbb{R})$. 


\section{ACKNOWLEDGMENTS}

I would like to thank Dr. Chan for his unwavering patience and keen guidance. His relaxed nature and abundant kindness made the whole process truly enjoyable. His prowess as a speaker is parallel to none, as he can make even the most complicated argument understandable. He is a true master, and none of this would be possible without him.

I would also like to thank Dr. Seubert, who has had a profound impact on my teaching philosophy and practice. His passion for teaching students to think and his ability to convey the bigger picture are contagious. I hope to emulate his passion in the classroom and show my future students that what we are training them to do matters in the real world.

I have made some great friends along the way. Shout outs, in no particular order, to Jake Laubacher, Kevin Stoll, Mike Conwood, Jen Magee, Todd Romutis, Mark Medwid, Dimitris Papathanasiou, Sam Carolus, Leo Pinheiro, Rob Kelvey, and anyone I am forgetting at this moment.

Lastly, I would like to thank my family: my parents for their advice, my wife Marie for her constant support and ability to put up with me, my brother Ted and his family, especially my two

favorite people in the world, Noelle and Colette. It was always nice to be brought back to reality now and again to be reminded of what truly makes me happy. 
TABLE OF CONTENTS

Page

CHAPTER 1 INTRODUCTION TO UNIVERSALITY $\ldots \ldots \ldots \ldots \ldots$. . . . . . . . . 1

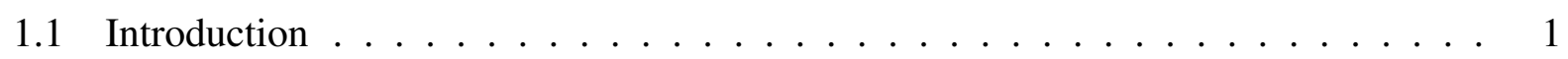

1.2 Motivation . . . . . . . . . . . . . . . . . . . 3

1.3 Main Result and Outline of Dissertation $\ldots \ldots \ldots \ldots$

CHAPTER 2 UNIVERSALITY CRITERIA $\ldots \ldots \ldots \ldots \ldots$

2.1 Two Universality Criteria for Semigroup Homomorphisms $\ldots \ldots$. . . . . . . . . 9

CHAPTER 3 UNIVERSALITY ON THE BALL OF BOUNDED ANALYTIC FUNC-

TIONS ON THE OPEN UNIT DISK $\ldots \ldots \ldots$. . . . . . . . . . . . . 17

3.1 Introduction . . . . . . . . . . . . . . . . . . 17

3.2 The Topology of Compact Convergence $\ldots \ldots \ldots \ldots$

3.3 Blaschke Products . . . . . . . . . . . . . . . . . . . . . . 23

3.4 Singular Inner Functions $\ldots \ldots \ldots \ldots$

3.5 Inner Functions in Several Variables $\ldots \ldots \ldots$. . . . . . . . . . 35

CHAPTER 4 UNIVERSALITY ON THE CLOSED UNIT BALL OF THE OPERATOR ALGEBRA OF CLASSICAL BANACH SPACES . . . . . . . . . . . . . . . . . . 39

4.1 Introduction $\ldots \ldots \ldots \ldots \ldots$

4.2 The Strong Operator Topology $\ldots \ldots$. . . . . . . . . . . . . . . . . . . . . . . . . 39

4.3 Universality of Conjugation Maps $\ldots \ldots \ldots \ldots \ldots$

CHAPTER 5 UNIVERSALITY ON THE CLOSED UNIT BALL OF BOUNDED MEA-

SURABLE FUNCTIONS $\ldots \ldots \ldots \ldots$. . . . . . . . . . . . . . . . . . . 49 
CHAPTER 6 UNIVERSALITY ON THE BALL OF ALL BOUNDED AND INTEGRABLE

MEASURABLE FUNCTIONS $\ldots \ldots \ldots \ldots \ldots$

CHAPTER 7 FUTURE WORK $\ldots \ldots \ldots \ldots \ldots \ldots$

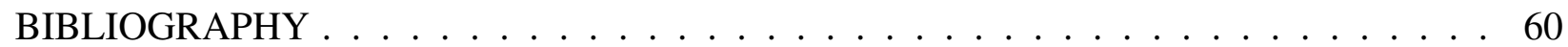




\section{CHAPTER 1 INTRODUCTION TO UNIVERSALITY}

\subsection{Introduction}

A sequence of continuous maps $\left\{T_{n}: X \rightarrow X \mid n \geq 1\right\}$ on a separable, metrizable topological space $X$ is said to be universal if there is an element $x$ in $X$ such that the set $\left\{T_{n} x: n \geq 1\right\}$ is dense in $X$. Such an element $x$ is called a universal element of the sequence $\left\{T_{n}\right\}$. When each member $T_{n}$ of a universal sequence is the $n$th power $T^{n}$ of a particular continuous map $T: X \rightarrow X$, then $T$ is said the be hypercyclic, and $x$ is called a hypercyclic element of $T$.

Some of the earliest examples of universality were exhibited on the vector space $H(G)$ of all analytic functions on a region $G$ in the complex plane $\mathbb{C}$. We recall that an $F$-space is a vector space $V$ with a metric $d$ that satisfies the following:

1. scalar multiplication and vector addition are continuous with resprect to $d$,

2. $d$ is translation invariant, meaning $d(x+z, y+z)=d(x, y)$ for all $x, y, z \in V$, and

3. the metric space $(V, d)$ is complete.

Given the topology of compact convergence, $H(G)$ becomes an $F$-space, in which a sequence $\left\{f_{n}\right\}$ converges to $f$ if and only if $f_{n} \rightarrow f$ uniformly on compact subsets of $G$. When the region $G=\mathbb{C}$, one of the very first examples of universality came when Birkhoff [5] showed in 1929 that the sequence of translation maps

$$
T_{n}: f(z) \mapsto f(z+n)
$$

is universal. When $G$ is the open unit disk $\mathbb{D}$, Seidel and Walsh [22] showed in 1941 that if $\left\{a_{n}\right\}$ is a sequence of points in $\mathbb{D}$ with $a_{n} \rightarrow 1$, and if

$$
\phi_{n}(z)=\frac{a_{n}-z}{1-\overline{a_{n}} z}
$$


then the sequence of non-Euclidean translations

$$
T_{n}: f(z) \mapsto f \circ \phi_{n}(z)
$$

is universal. The next example of universality came in 1952, when MacLane [17] showed that the sequence of derivative maps

$$
D_{n}: f(z) \mapsto f^{(n)}(z)
$$

is universal on $H(\mathbb{C})$. The first example of universality on a Banach space was given by Rolewicz [20] in 1969, when he showed that any scalar multiple of the backward shift $B$ given by

$$
T=\lambda B:\left(x_{1}, x_{2}, x_{3}, \ldots\right) \mapsto \lambda\left(x_{2}, x_{3}, x_{4}, \ldots\right)
$$

with $|\lambda|>1$ is hypercyclic on any $\ell^{p}$ space where $1 \leq p<\infty$.

These four results were given a unified approach in the 1987 landmark paper of Gethner and Shapiro [11], who provided a sufficient condition for universality on an $F$-space, which is now known as the Universality Criterion. Their sufficient condition is a refined version of Kitai [16], and it birthed the systematic study of universality by uniting all known examples at the time. We state it here.

Theorem 1.1. Let $\left\{T_{n}: X \rightarrow X \mid n \geq 1\right\}$ be a sequence of continuous linear operators on an F-space $X$. Let $\mathcal{D}_{1}, \mathcal{D}_{2}$ be two dense subsets of $X$, and $\left\{A_{n}: \mathcal{D}_{2} \rightarrow X \mid n \geq 1\right\}$ be a sequence of mappings. Suppose

(i) $T_{n} g \rightarrow 0$, for every $g \in \mathcal{D}_{1}$.

(ii) $A_{n} f \rightarrow 0$, for every $f \in \mathcal{D}_{2}$.

(iii) $T_{n} A_{n} f \rightarrow f$, for every $f \in \mathcal{D}_{2}$.

Then $\left\{T_{n}\right\}$ has a dense $G_{\delta}$ of universal elements. 
The original proofs of the examples mentioned above were all constructive in nature; that is, a particular universal element was exhibited for the situation at hand. Kitai's proof of the criterion was constructive, but only applied in the Banach space setting. Gethner and Shapiro's approach works in the more general setting of an F-space, but does not provide a specific form of the universal element $x$ in $X$ for the universal sequence $\left\{T_{n}\right\}$. In Corollary 2.2 below, we provide a constructive approach that generalizes Gethner and Shapiro's Universality Criterion from an Fspace to a more general setting of a separable, metrizable topological semigroup $X$ with identity $e$. Before going in to details, we mention a motivating example.

\subsection{Motivation}

In response to a question from MacLane, Heins [15] showed in 1955 the existence of a Blaschke product $B$ such that, if $\left\{a_{n}\right\}$ is a sequence of points in $\mathbb{D}$ with $a_{n} \rightarrow 1$, and if

$$
\phi_{n}(z)=\frac{a_{n}-z}{1-\overline{a_{n}} z}
$$

then the sequence of non-Euclidean translations of $B$ given by

$$
\left\{B \circ \phi_{1}, B \circ \phi_{2}, B \circ \phi_{3}, \ldots\right\}
$$

is dense in the set $X=\left\{f \in H^{\infty}(\mathbb{D}):\|f\|_{\infty} \leq 1\right\}$ with the topology of compact convergence. Recall that a Blaschke product is a convergent product of disk automorphisms, and thus can be written as

$$
B(z)=\prod_{k=1}^{\infty} \frac{\overline{a_{k}}}{\left|a_{k}\right|} \frac{a_{k}-z}{1-\overline{a_{k}} z}
$$

where $a_{k} \in \mathbb{D}$ and

$$
\sum_{k=1}^{\infty}\left(1-\left|a_{k}\right|\right)<\infty .
$$

Heins' result cannot be deduced from Gethner and Shapiro's Universality Criterion for two main reasons. First, the set $X$ is not an $F$-space since it is not a vector space; $X$ is not closed 
under addition. Second, Heins' result proves a universal element of a certain form exists, namely a Blaschke product, and the result of Gethner and Shapiro does not construct a particular universal element.

However, the set $X$ does share many nice properties with a general $F$-space. With the topology of compact convergence, $X$ becomes a metrizable topological space (see Section 3.2). While $X$ is not closed under function addition, it is closed under function multiplication. Furthermore, multiplication is continuous with respect to this metric, which means that if $\left\{f_{n}\right\}$ and $\left\{g_{n}\right\}$ are sequences in $X$ with $f_{n} \rightarrow f$ and $g_{n} \rightarrow g$, then

$$
f_{n} g_{n} \rightarrow f g
$$

Moreover, $X$ is a complete metric space with the topology of compact convergence. Thus $X$ has similar qualities to conditions (1) and (3) in the definition of an F-space, where the main difference is that vector addition has been replaced with the associative binary operation of multiplication of functions. A vector space also has an additive identity element, namely the 0 vector. The set $X$ contains a multiplicative identity element, namely the constant function 1 . Also, the operation of multiplication of functions is commutative, but this is a superfluous feature of the operation for our purposes. These properties of $X$ make $X$ a complete, metrizable, topological semigroup with an identity element. It is the example to keep in mind throughout the next section as we proceed in a more formal manner.

Before we state precise definitions of the terms above, we end this section by mentioning that the analogue for $X$ of a continuous linear operator on an $F$-space is a continuous multiplicative mapping from $X$ into $X$. When considering a vector space, a linear mapping of the vector space into itself is a homomorphism since it preserves the underlying operation of the space, which is vector addition. Since the underlying operation defined on $X$ is function multiplication, we would like to consider continuous maps that preserve multiplication. The composition operators 
$\left\{T_{n}: X \rightarrow X\right\}$ defined by

$$
T_{n}: f(z) \mapsto f \circ \phi(z)
$$

are multiplicative homomorphisms, since

$$
T_{n}(f g)=(f g) \circ \phi=(f \circ \phi)(g \circ \phi)
$$

We now look at these notions in full generality, keeping in mind that our goal is to use our general Corollary 2.2 to prove Heins' result and its analogues, as well as provide new applications.

\subsection{Main Result and Outline of Dissertation}

In this section, we mention our main result and how the rest of the dissertation is structured around it. To state our result, we offer a few definitions. A semigroup is a set $X$ together with an associative binary operation from $X \times X$ into $X$. A homomorphism on $X$ is a map from $X$ into $X$ that preserves the binary operation. A semigroup $X$ is a topological semigroup if $X$ carries a topology with respect to which its binary operation is continuous. For a semigroup $X$, we represent the operation as multiplication. The main result of this dissertation is Corollary 2.2 below, which provides the following generalization of Gethner and Shapiro's Universality Criterion for a sequence of continuous homomorphisms $\left\{T_{n}\right\}$ on a separable, complete, metrizable, topological semigroup $X$ with identity $e$ :

suppose $\mathcal{D}_{1}, \mathcal{D}_{2}$ are two dense subsets of $X$, and $\left\{A_{n}: \mathcal{D}_{2} \rightarrow X \mid n \geq 1\right\}$ is a sequence of mappings satisfying conditions

(i) $T_{n} g \rightarrow e$, for every $g \in \mathcal{D}_{1}$.

(ii) $A_{n} f \rightarrow e$, for every $f \in \mathcal{D}_{2}$.

(iii) $T_{n} A_{n} f \rightarrow f$, for every $f \in \mathcal{D}_{2}$. 
Then $\left\{T_{n}\right\}$ has a dense $G_{\delta}$ set of universal elements, and there is a universal element $x$ in $X$ of the form

$$
x=\prod_{j=1}^{\infty} g_{j},
$$

where each $g_{j}$ is an element of $\mathcal{D}_{1}$.

By taking the semigroup $X$ in Corollary 2.2 to be a Fréchet space $X$, which is an additive group, and the semigroup identity to be the zero vector of $X$, Corollary 2.2 provides a universal element $x$ that works for Gethner and Shaprio's Criterion. Since Kitai's construction takes place in a Banach space and makes heavy use of linearity, neither her nor Gethner and Shapiro's methods work in our setting. Consequently we need to construct a universal element $x$ in the semigroup $X$ using the topological and algebraic properties of the semigroup. Motivated by our Corollary 2.2. we provide Theorem 2.1 which further generalizes the constructive approach to the setting where $X$ is a semigroup without identity and its topology does not provide the continuity of the semigroup multiplication. In fact, we prove Theorem 2.1 in the general setting first and then use it to prove Corollary 2.2 as a corollary.

To provide applications of Corollary 2.2, in Chapter 3 we take $X$ to be the multiplicative semigroup of the closed unit ball of the algebra $H^{\infty}(\mathbb{D})$ of all bounded analytic functions on $\mathbb{D}$. Directly applying Corollary 2.2, we provide simple proofs for a few generalizations of the classical result of Heins [15] in 1955 who showed that that if $a_{n}$ and $\phi_{n}$ are as given in Seidel and Walsh's result, then there exists a universal Blaschke product $B$ so that the set

$$
\left\{B, B \circ \phi_{1}, B \circ \phi_{2}, B \circ \phi_{3}, \ldots\right\}
$$

is dense in $X$, with the topology of compact convergence. In Theorem 3.16, we use Corollary 2.2 to obtain a simple proof for a 2004 result of Gorkin and Mortini [13], who proved that under the same hypothesis on $a_{n}$ and $\phi_{n}$, there is a singular inner function $S$ so that the set

$$
\left\{S, S \circ \phi_{1}, S \circ \phi_{2}, S \circ \phi_{3}, \ldots\right\}
$$


is dense, with respect to the topology of compact convergence, in the multiplicative semigroup of zero-free functions in the closed unit ball of the algebra $H^{\infty}(\mathbb{D})$ together with the constant zero function. Then we use Corollary 2.2 to give a simple proof of Theorem 3.20, which provides a simple form of a universal inner function of several variables that is analogous to Heins' onevariable result, improving a 2008 result of Bayart [2].

In Chapter 4 , we take $X$ to be closed unit ball of the operator algebra of $c_{0}$, or $\ell^{p}$ where $1 \leq p<\infty$. With the strong operator topology and the usual operator multiplication, $X$ is a separable, complete, metrizable topological semigroup whose identity is the identity operator $I$. In Theorem 4.8, we provide an infinite product of operators as a universal element for a sequence of conjugation maps $T_{n}$ defined by

$$
T_{n}(V)=U_{n}^{-1} V U_{n}
$$

where $U_{n}$ are isometric isomorphisms with $U_{n} \rightarrow 0$ and $U_{n}^{-1} \rightarrow 0$ in the weak operator topology.

In Chapters 5 and 6, we provide applications that require the generality of Theorem 2.1. First in Chapter 5, we take $X$ to be closed unit ball of the Banach algebra $L^{\infty}[0,1]$ of all bounded Lebesgue measurable functions. With the weak-star topology, $X$ becomes a semigroup that is compact, metrizable, and complete, but $X$ is not a topological semigroup. Nevertheless, we can use Theorem 2.1 to provide a universal element for the sequence of composition operators $T_{n}$ on $X$ defined by

$$
T_{n} f=f \circ \phi_{n}
$$

where $\phi_{n}:[0,1] \rightarrow[0,1]$ are strictly increasing functions with $\phi_{n} \rightarrow 1$ pointwise on $[0,1]$.

In Chapter 6, we take $X$ to be the semigroup $\left\{f \in L^{\infty}(\mathbb{R}):\|f\|_{\infty} \leq 1\right.$ and $\|f\|_{1}=\int|f| d m<$ $\infty\}$, where $m$ is the Lebesgue measure. With the $\|\cdot\|_{1}$-topology and the usual multiplication of functions, $X$ becomes a separable, complete, metrizable topological semigroup without an identity because the constant function 1 does not belong to $X$. Before stating our application of Theorem 2.1 on $X$, we first give a definition. A function $\psi: \mathbb{R} \rightarrow \mathbb{R}$ is called a staircase function if there exist sequences $\left\{a_{n}:-\infty<n<\infty\right\}$ and $\left\{c_{n}:-\infty<n<\infty\right\}$ of strictly increasing real 
numbers such that both sequences are not bounded above nor below, and

$$
\psi=\sum_{n=-\infty}^{\infty} a_{n} \chi_{\left(c_{n}, c_{n+1}\right)} .
$$

We can use Theorem 2.1 to show that if $\phi_{n}: \mathbb{R} \rightarrow \mathbb{R}$ are continuous, surjective, strictly increasing functions converging almost everywhere to a staircase function, and for which the corresponding composition maps $T_{n}: X \rightarrow X$ given by

$$
T_{n} f=f \circ \phi_{n}
$$

are continuous, then the sequence of operators $\left\{T_{n}\right\}$ is universal. Lastly, we conclude our discussion with some other universality results for $C(\mathbb{R})$ and $L^{1}(\mathbb{R})$. 


\section{CHAPTER 2 UNIVERSALITY CRITERIA}

\subsection{Two Universality Criteria for Semigroup Homomorphisms}

In this section we construct a universal element in a separable, complete, metrizable topological semigroup $X$ for a sequence of continuous homomorphisms on $X$ satisfying the well-known universality criterion which was first obtained by Chan [6] and later by Moothathu [23].

In Theorem 2.1 below, we construct a universal element without the assumption that the semigroup $X$ is a topological semigroup, nor the assumption that $X$ possess an identity element. Then in Corollary 2.2, we show how those two assumptions reduce Theorem 2.1 to the format of the universality criterion obtained in [6] and [23].

Theorem 2.1. Suppose $X$ is a separable, complete, metrizable semigroup such that for any $g \in X$, the left multiplication by $g$ on $X$ is continuous, and $\left\{T_{n}: X \rightarrow X \mid n \geq 1\right\}$ is a sequence of continuous semigroup homomorphisms. If there exist a dense semigroup $\mathcal{D}_{1}$ of $X$, a dense subset $\mathcal{D}_{2}$ of $X$ and a sequence of mappings $\left\{A_{n}: \mathcal{D}_{2} \rightarrow X \mid n \geq 1\right\}$ such that

1. $g\left(A_{n} f\right) \rightarrow g$, for every $g \in \mathcal{D}_{1}, f \in \mathcal{D}_{2}$, and

2. $\left(T_{n} g\right)\left(T_{n} A_{n} f\right)=T_{n}\left(g A_{n} f\right) \rightarrow f$, for every $g \in \mathcal{D}_{1}, f \in \mathcal{D}_{2}$,

then $\left\{T_{n}\right\}$ has a dense $G_{\delta}$ set of universal elements, and there is a universal element $x$ in $X$ of the form

$$
x=\prod_{j=1}^{\infty} g_{j}
$$

where each $g_{j}$ is an element of $\mathcal{D}_{1}$.

Proof. We first check that the set of all universal elements is a $G_{\delta}$ set given by $\bigcap_{k=1}^{\infty} \bigcup_{n=1}^{\infty} T_{n}^{-1} V_{k}$, where $\left\{V_{k}: k \geq 1\right\}$ is a base of the topology of $X$. First, suppose $x$ is a universal element for $\left\{T_{n}\right\}$. Let $V_{k}$ be any basis element of the topology of $X$, and let $y \in V_{k}$. Since $V_{k}$ is open, there is 
a number $\epsilon>0$ for which the set

$$
B_{\epsilon}(y)=\{z \in X: d(z, y)<\epsilon\}
$$

is contained in $V_{k}$. By the density of the sequence $\left\{T_{n} x\right\}$ in $X$, there is some $n$ for which $d\left(T_{n} x, y\right)<\epsilon$. Thus $T_{n} x \in B_{\epsilon}(y)$, so $T_{n} x \in V_{k}$. Hence the set of universal elements for $\left\{T_{n}\right\}$ is contained in $\bigcap_{k=1}^{\infty} \bigcup_{n=1}^{\infty} T_{n}^{-1} V_{k}$.

Conversely, suppose $x \in \bigcap_{k=1}^{\infty} \bigcup_{n=1}^{\infty} T_{n}^{-1} V_{k}$. Let $y \in X$ and $\epsilon>0$ be given. Since $B_{\epsilon}(y)$ is open, there is some basis element $V_{k}$ for which $y \in V_{k}$ and $V_{k} \subset B_{\epsilon}(y)$. For this $V_{k}$, there is some $n$ for which

$$
x \in T_{n}^{-1} V_{k}
$$

Thus $T_{n} x \in V_{k}$, so $d\left(T_{n} x, y\right)<\epsilon$. Hence the sequence $\left\{T_{1} x, T_{2} x, T_{3} x, \ldots\right\}$ is dense in $X$, and the set of all universal elements is the $G_{\delta}$ set $\bigcap_{k=1}^{\infty} \bigcup_{n=1}^{\infty} T_{n}^{-1} V_{k}$, finishing the proof of our claim.

To show $\left\{T_{n}\right\}$ has a dense $G_{\delta}$ set of universal elements by using the Baire Category Theorem, it suffices to prove that each open set $\bigcup_{n=1}^{\infty} T_{n}^{-1} V_{k}$ is dense. For that, we now prove that for each pair of nonempty open subsets $U$ and $V$ of $X$, there exists a positive integer $n$ such that $T_{n} U \cap V$ is nonempty.

Since the sets $\mathcal{D}_{1}$ and $\mathcal{D}_{2}$ in our hypothesis are dense, there exist elements $g \in \mathcal{D}_{1} \cap U$ and $f \in \mathcal{D}_{2} \cap V$. If we let $h_{n}=g A_{n} f$, then $h_{n} \rightarrow g$ and $T_{n} h_{n}=\left(T_{n} g\right)\left(T_{n} A_{n} f\right) \rightarrow f$ by hypotheses (1) and (2), respectively. It follows that if $n$ is sufficiently large, then $h_{n} \in U$ and $T_{n} h_{n} \in V$.

We now construct a particular universal element $x=\prod_{j=1}^{\infty} g_{j}$ using an inductive process to choose the factors $g_{1}, g_{2}, \ldots$ In this proof we use $d$ to denote a metric giving the topology of $X$.

Since $X$ is separable, $\mathcal{D}_{2}$ contains a dense sequence $\left\{f_{1}, f_{2}, \ldots\right\}$. Pick any element $g_{0} \in \mathcal{D}_{1}$. Since $T_{n}\left(g_{0} A_{n} f_{1}\right) \rightarrow f_{1}$ by hypothesis (2), choose $n_{1}$ such that

$$
d\left(T_{n_{1}}\left(g_{0} A_{n_{1}} f_{1}\right), f_{1}\right)<2^{-2}
$$


Then, using the continuity of $T_{n_{1}}$ at $g_{0} A_{n_{1}} f_{1}$, choose $g_{1}$ from $\mathcal{D}_{1}$ such that

$$
d\left(g_{0} A_{n_{1}} f_{1}, g_{1}\right)<2^{-2} \text { and } d\left(T_{n_{1}} g_{1}, T_{n_{1}}\left(g_{0} A_{n_{1}} f_{1}\right)\right)<2^{-2} .
$$

Hence by (2.1.1) we have

$$
\begin{aligned}
& d\left(T_{n_{1}} g_{1}, f_{1}\right) \\
\leq & d\left(T_{n_{1}} g_{1}, T_{n_{1}}\left(g_{0} A_{n_{1}} f_{1}\right)\right)+d\left(T_{n_{1}}\left(g_{0} A_{n_{1}} f_{1}\right), f_{1}\right)<2^{-1} .
\end{aligned}
$$

Inductively suppose for $k \geq 2$, we have found increasing integers $n_{1}<n_{2}<\cdots<n_{k-1}$ and elements $g_{1}, g_{2}, \cdots, g_{k-1}$ in $\mathcal{D}_{1}$. We now define the integer $n_{k}$ and the element $g_{k}$ as follows.

Let $x_{k-1}=\prod_{j=1}^{k-1} g_{j}$. By the continuity of $T_{n_{1}}, T_{n_{2}}, \ldots, T_{n_{k-1}}$ at $x_{k-1}$, there exists a positive $\delta$ such that if $1 \leq j \leq k-1$, and if $y$ is an element in $X$ with $d\left(y, x_{k-1}\right)<\delta$, then

$$
d\left(T_{n_{j}} y, T_{n_{j}} x_{k-1}\right)<2^{-(k+2)} .
$$

Since $x_{k-1}$ is in $\mathcal{D}_{1}$ and $f_{k}$ is in $\mathcal{D}_{2}$, choose $n_{k}$ so that we have, by hypotheses (1) and (2) respectively,

$$
\begin{aligned}
& d\left(x_{k-1} A_{n_{k}} f_{k}, x_{k-1}\right)<2^{-1} \min \left\{2^{-k-1}, \delta\right\}, \text { and } \\
& d\left(T_{n_{k}}\left(x_{k-1} A_{n_{k}} f_{k}\right), f_{k}\right)<2^{-k-2} .
\end{aligned}
$$

We are ready to move on to define $g_{k}$. Since $T_{n_{k}}$ is continuous at $x_{k-1} A_{n_{k}} f_{k}$, there is a positive $\rho$ such that if $y$ is an element in $X$ with $d\left(y, x_{k-1} A_{n_{k}} f_{k}\right)<\rho$, then

$$
d\left(T_{n_{k}} y, T_{n_{k}}\left(x_{k-1} A_{n_{k}} f_{k}\right)\right)<2^{-k-2} .
$$


Also, by our hypotheses, the left multiplication by $x_{k-1}$ is continuous on $X$, so there exists a positive $\gamma$ such that if $y$ is an element in $X$ with $d\left(y, A_{n_{k}} f_{k}\right)<\gamma$ then

$$
d\left(x_{k-1} y, x_{k-1} A_{n_{k}} f_{k}\right)<2^{-1} \min \left\{2^{-k-3}, \delta, \rho\right\}
$$

Choose $g_{k}$ in $\mathcal{D}_{1}$ so that

$$
d\left(g_{k}, A_{n_{k}} f_{k}\right)<\gamma
$$

Then by (2.1.8) and (2.1.5),

$$
\begin{aligned}
& d\left(x_{k-1} g_{k}, x_{k-1}\right) \\
\leq & d\left(x_{k-1} g_{k}, x_{k-1} A_{n_{k}} f_{k}\right)+d\left(x_{k-1} A_{n_{k}} f_{k}, x_{k-1}\right) \\
< & \min \left\{2^{-k-1}, \delta\right\} .
\end{aligned}
$$

Thus whenever $1 \leq j \leq k-1$, inequality 2.1.4 implies that

$$
d\left(T_{n_{j}}\left(x_{k-1} g_{k}\right), T_{n_{j}} x_{k-1}\right)<2^{-(k+2)} .
$$

We now claim that

$$
d\left(T_{n_{k}}\left(x_{k-1} g_{k}\right), f_{k}\right)<2^{-k-1}
$$

For that we use the triangle inequality to write

$$
d\left(T_{n_{k}}\left(x_{k-1} g_{k}\right), f_{k}\right) \leq d\left(T_{n_{k}}\left(x_{k-1} A_{n_{k}} f_{k}\right), f_{k}\right)+d\left(T_{n_{k}}\left(x_{k-1} g_{k}\right), T_{n_{k}}\left(x_{k-1} A_{n_{k}} f_{k}\right)\right)
$$

The first summand in the previous expression is less than $2^{-k-2}$ by 2.1 .6 . For the second summand, we observe that since $d\left(g_{k}, A_{n_{k}} f_{k}\right)<\gamma$ by (2.1.9), we have $d\left(x_{k-1} g_{k}, x_{k-1} A_{n_{k}} f_{k}\right)<\rho$ by 
2.1.8). Thus by (2.1.7),

$$
d\left(T_{n_{k}}\left(x_{k-1} g_{k}\right), T_{n_{k}}\left(x_{k-1} A_{n_{k}} f_{k}\right)\right)<2^{-k-2},
$$

which shows our claim (2.1.12).

Having inductively defined a sequence of increasing integers $\left\{n_{k}\right\}$ and a sequnce of elements $\left\{g_{k}\right\}$ in $\mathcal{D}_{1}$, we let

$$
x_{k}=\prod_{j=1}^{k} g_{j} \text {. }
$$

We claim that $\left\{x_{k}\right\}$ is a Cauchy sequence. This follows from the fact that if $m>k$ then by 2.1 .10 ,

$$
\begin{aligned}
& d\left(x_{k}, x_{m}\right) \\
\leq & d\left(x_{k}, x_{k+1}\right)+d\left(x_{k+1}, x_{k+2}\right)+\cdots+d\left(x_{m-1}, x_{m}\right) \\
\leq & 2^{-(k+2)}+2^{-(k+3)}+\cdots \\
= & 2^{-(k+1)} .
\end{aligned}
$$

Thus we can define an element $x$ as the infinite product

$$
x=\prod_{j=1}^{\infty} g_{j} .
$$

We now complete the proof by showing that $x$ is a universal element of $\left\{T_{n}\right\}$, using the following computations.

$$
\begin{aligned}
& d\left(T_{n_{k}} x, f_{k}\right) \\
\leq & d\left(T_{n_{k}} x, T_{n_{k}} x_{k}\right)+d\left(T_{n_{k}} x_{k}, f_{k}\right) .
\end{aligned}
$$


For the first summand in the previous expression, we use 2.1.11) to see that

$$
\begin{aligned}
& d\left(T_{n_{k}} x, T_{n_{k}} x_{k}\right) \\
= & \lim _{m \rightarrow \infty} d\left(T_{n_{k}} x_{m}, T_{n_{k}} x_{k}\right) \\
\leq & \lim _{m \rightarrow \infty} \sum_{j=k}^{m-1} d\left(T_{n_{k}} x_{j}, T_{n_{k}} x_{j+1}\right) \\
\leq & \lim _{m \rightarrow \infty} \sum_{j=k}^{m-1} 2^{-(j+2)} \\
\leq & \sum_{j=k}^{\infty} 2^{-(j+2)}=2^{-k-1} .
\end{aligned}
$$

For the second summand in 2.1.15), inequality 2.1.12) implies that it is less than $2^{-k-1}$. Combining the estimations on the two summands we have

$$
d\left(T_{n_{k}} x, f_{k}\right) \leq 2^{-k}
$$

finishing the whole proof by the denseness of the sequence $\left\{f_{j}\right\}$.

Theorem 2.1 is applied in Chapter 5 to a semigroup which is not a topological semigroup, and in Chapter 6 to a topological semigroup that has no identity element. In the case that $X$ is a topological semigroup with identity element $e$, we can rephrase Theorem 2.1 with stronger hypotheses which are parallel to the usual universality criterion, as stated in the following Corollary 2.2

Note that the two dense sets $\mathcal{D}_{1}$ and $\mathcal{D}_{2}$ in Theorems 2.1 and 2.2 are not assumed to be the same. A few remarks about $\mathcal{D}_{1}$ and $\mathcal{D}_{2}$ are in order, for the case that $T_{n}$ are bounded linear operators on a Banach space $X$. When $\mathcal{D}_{1}=\mathcal{D}_{2}$, Kitai [16] showed a way to construct a hypercyclic vector. However when $\mathcal{D}_{1} \neq \mathcal{D}_{2}$, Gethner and Shapiro [11] showed the existence of hypercyclic vectors as a result of the Baire Category Theorem. Nevertheless, we demonstrate in Corollary 2.2 below that a universal element can indeed be still constructed even when $\mathcal{D}_{1} \neq \mathcal{D}_{2}$ and $X$ is merely a 
semigroup. Then we show in the next few sections our constructive approach for giving the special form of a universal element is important in obtaining classical results as well as new results. One particular application in which $\mathcal{D}_{1} \neq \mathcal{D}_{2}$ is provided by Theorem 3.20 .

Corollary 2.2. Let $X$ be a separable, complete, metrizable, topological semigroup with identity e, and $\left\{T_{n}: X \rightarrow X \mid n \geq 1\right\}$ be a sequence of continuous semigroup homomorphisms. Let $\mathcal{D}_{1}, \mathcal{D}_{2}$ be two dense subsets of $X$, and $\left\{A_{n}: \mathcal{D}_{2} \rightarrow X \mid n \geq 1\right\}$ be a sequence of mappings. Suppose

(i) $T_{n} g \rightarrow e$, for every $g \in \mathcal{D}_{1}$.

(ii) $A_{n} f \rightarrow e$, for every $f \in \mathcal{D}_{2}$.

(iii) $T_{n} A_{n} f \rightarrow f$, for every $f \in \mathcal{D}_{2}$.

Then $\left\{T_{n}\right\}$ has a dense $G_{\delta}$ set of universal elements, and there is a universal element $x$ in $X$ of the form

$$
x=\prod_{j=1}^{\infty} g_{j},
$$

where each $g_{j}$ is an element of $\mathcal{D}_{1}$.

Proof. Since hypothesis (i) of our Corollary implies that $T_{n} g \rightarrow e$ for any $g$ in the semigroup generated by $\mathcal{D}_{1}$, we may assume that $\mathcal{D}_{1}$ is a dense semigroup. To prove our Corollary, we now verify hypotheses (1) and (2) of Theorem 2.1 are satisfied. Let $g$ be in $\mathcal{D}_{1}$ and $f$ be in $\mathcal{D}_{2}$. By assumption, $A_{n} f \rightarrow e$. Hence $g A_{n} f \rightarrow g e=g$ by the continuity of multiplication. Similarly, since $T_{n} g \rightarrow e$ and $T_{n} A_{n} f \rightarrow f$, the continuity of multiplication yields that $\left(T_{n} g\right)\left(T_{n} A_{n} f\right) \rightarrow e f=f$. Thus the hypotheses of Theorem 2.1 are satisfied, and the proof is complete.

The usual form of the universality criterion is stated in the setting of a Fréchet space. Of course, a Fréchet space is a topological vector space. Thus we can view a Fréchet space as a topological semigroup whose binary operation is addition and whose identity element is the zero vector. There are two noteworthy differences between Theorem 2.1 and any typical version of the universality criterion for a Fréchet space. First, the semigroup $X$ need not have an identity element. Second, continuity of the binary operation from $X \times X$ into $X$ is not required, where 
instead we only assume the left multiplication by a fixed element is continuous. The trade-off is that the convergence conditions in hypotheses (1) and (2) require more care to verify.

In Chapters 3 and 4 below, we give applications of Corollary 2.2 on semigroups with identity elements, while in Chapters 5 and 6 we provide examples of universality where the more general setting of Theorem 2.1 is required. 


\section{CHAPTER 3 UNIVERSALITY ON THE BALL OF BOUNDED ANALYTIC FUNCTIONS ON THE OPEN UNIT DISK}

\subsection{Introduction}

In this chapter, we apply our constructive approach in Corollary 2.2 to simplify the proofs of a few results in the literature concerning analytic functions defined on the open unit ball $\mathbb{B}_{N}=\{z \in$ $\left.\mathbb{C}^{N}:\|z\|<1\right\}$ of $\mathbb{C}^{N}$, where $N \geq 1$, with the topology of compact convergence. In the special case when $N=1$, the ball $\mathbb{B}_{N}$ becomes the open unit disk $\mathbb{D}=\{z \in \mathbb{C}|| z \mid<1\}$, for which the inner functions are divided into 2 types, the Blaschke products and the singular inner functions.

By taking the semigroup in Corollary 2.2 to be the closed unit ball $X=\overline{\operatorname{Ball}}\left(H^{\infty}(\mathbb{D})\right)=$ $\left\{f \in H^{\infty}(\mathbb{D}):\|f\|_{\infty} \leq 1\right\}$ of bounded analytic functions on $\mathbb{D}$, we focus on the universality of Blaschke products in Section 3.3 below. Then in Section 3.4, we take the semigroup in Corollary 2.2 to be $\mathcal{N}=\{f \in X: f(z) \neq 0$ for all $z \in \mathbb{D}\} \cup\{0\}$ and study the universality of singular inner functions. Lastly in Section 3.5, to obtain the $N$-variable generalizations of our results, we take the semigroup in Corollary 2.2 to be the closed unit ball $\mathcal{B}=\left\{f \in H^{\infty}\left(\mathbb{B}_{N}\right):\|f\|_{\infty} \leq 1\right\}$ of the bounded analytic functions on $\mathbb{B}_{N}$. But first, we discuss the topology of compact convergence on the set $X$ to show that $X$ is a complete, metrizable, topological semigroup.

\subsection{The Topology of Compact Convergence}

Our goal in this section is to define a topology on the semigroup $X=\left\{f \in H^{\infty}(\mathbb{D}):\|f\|_{\infty} \leq\right.$ 1 ) and show that $X$ is a complete, metrizable topological semigroup when endowed with this topology. We begin by recalling some terminology.

If $S$ is a set, a basis for a topology on $S$ is a collection $\mathcal{A}$ of subsets of $X$ such that

(1) For each $f \in S$, there is at least one basis element $B$ containing $f$, and

(2) If $f$ belongs to the intersection of two basis elements $B_{1}$ and $B_{2}$, then there is a basis element $B_{3}$ containing $f$ such that $B_{3} \subset B_{1} \cap B_{2}$. 
The topology generated by a basis $\mathcal{A}$ is the collection of all unions of elements of $\mathcal{A}$ (see [19, Lemma 13.1]).

Given elements $f, g \in X$, a compact set $C \subset \mathbb{D}$ and a number $\epsilon>0$, let

$$
\|f-g\|_{C}=\sup _{z \in C}|f(z)-g(z)|
$$

and let

$$
B_{C}(f, \epsilon)=\left\{g \in X:\|f-g\|_{C}<\epsilon\right\}
$$

The sets $B_{C}(f, \epsilon)$ form a basis for a topology on $X$, called the topology of compact convergence. We quickly check that the sets $B_{C}(f, \epsilon)$ indeed form a basis.

Lemma 3.1. The sets $B_{C}(f, \epsilon)$ form a basis.

Proof. Let $g \in X$ be given. Since $g \in B_{C}(g, \epsilon)$ for any compact set $C \subset \mathbb{D}$ and any $\epsilon>0$, condition (1) of a basis is trivially satisfied. To check condition (2), suppose there are $f_{1}, f_{2} \in X$, compacts sets $C_{1} \subset \mathbb{D}$ and $C_{2} \subset \mathbb{D}$, and numbers $\epsilon_{1}>0, \epsilon_{2}>0$ such that

$$
g \in B_{C_{1}}\left(f_{1}, \epsilon_{1}\right) \cap B_{C_{2}}\left(f_{2}, \epsilon_{2}\right) .
$$

Let

$$
\delta_{1}=\epsilon_{1}-\|f(z)-g(z)\|_{C_{1}}, \quad \delta_{2}=\epsilon_{2}-\|f(z)-g(z)\|_{C_{2}}
$$

let $C=C_{1} \cup C_{2}$ and let $\delta=\min \left\{\delta_{1}, \delta_{2}\right\}$. Then $B_{C}(g, \delta)$ is a basis element. We will show that

$$
B_{C}(g, \delta) \subset B_{C_{1}}\left(f_{1}, \epsilon_{1}\right) \cap B_{C_{2}}\left(f_{2}, \epsilon_{2}\right) .
$$

Let $h \in B_{C}(g, \delta)$. We compute that

$$
\begin{aligned}
\left\|f_{1}(z)-h(z)\right\|_{C_{1}} & \leq\left\|f_{1}(z)-g(z)\right\|_{C_{1}}+\|g(z)-h(z)\|_{C_{1}} \\
& \leq \delta+\epsilon_{1}-\delta=\epsilon_{1} .
\end{aligned}
$$


Thus $h \in B_{C_{1}}\left(f_{1}, \epsilon_{1}\right)$. Similarly, we compute that

$$
\begin{aligned}
\left\|f_{2}(z)-h(z)\right\|_{C_{1}} & \leq\left\|f_{1}(z)-g(z)\right\|_{C_{1}}+\|g(z)-h(z)\|_{C_{1}} \\
& \leq \delta+\epsilon_{1}-\delta=\epsilon_{1} .
\end{aligned}
$$

Thus $h \in B_{C_{1}}\left(f_{1}, \epsilon_{1}\right) \cap B_{C_{2}}\left(f_{2}, \epsilon_{2}\right)$, and the proof is complete.

As is often the case, it is easier to work with a notion of convergent sequences rather than working with a basis for a topology. An immediate consequence of the topology of compact convergence, and a justification for its name, comes from the fact that a sequence of functions $\left\{f_{n}\right\}$ in $X$ converges to $f$ in the topology of compact convergence if and only if for each compact set $C \subset \mathbb{D}$, the sequence $\left\{f_{n}\right\}$ converges to $f$ uniformly on $C$.

We define a metric on $X$ as follows: take an exhaustion of $\mathbb{D}$ by compact sets $\left\{C_{n}\right\}$, that is, compact sets $C_{n}$ satisfying

(i) $C_{n}$ is contained in the interior of $C_{n+1}$, and

(ii) $\mathbb{D}=\bigcup_{n=1}^{\infty} C_{n}$,

and define a metric $d$ by

$$
d(f, g)=\sum_{n=1}^{\infty} \frac{1}{2^{n}} \frac{\|f-g\|_{C_{n}}}{1+\|f-g\|_{C_{n}}} .
$$

The topology induced by the metric $d$ on $X$ is equal to the topology of compact convergence on $X$, which we prove in the following proposition.

Proposition 3.2. If $f_{k}, f \in X$, then $d\left(f_{k}, f\right) \rightarrow 0$ if and only if $\left\{f_{k}\right\}$ converges to $f$ uniformly on compact subsets.

Proof. First, suppose that $d\left(f_{k}, f\right) \rightarrow 0$, and let $C$ be compact and $\epsilon>0$ be given. Note that $\|f\|_{C_{n}} \leq\|f\|_{C_{n+1}}$ for all $n$ by the maximum modulus principle. Choose $\rho>0$ such that $\frac{1-\rho}{\rho}<\epsilon$ 
and choose $M$ such that

$$
d\left(f_{k}, f\right)=\sum_{n=1}^{\infty} \frac{1}{2^{n}} \frac{\left\|f_{k}-f\right\|_{C_{n}}}{1+\left\|f_{k}-f\right\|_{C_{n}}}<\rho
$$

for all $k \geq M$. Since

$$
\frac{\left\|f_{k}-f\right\|_{C_{n}}}{1+\left\|f_{k}-f\right\|_{C_{n}}} \leq \frac{\left\|f_{k}-f\right\|_{C_{n+1}}}{1+\left\|f_{k}-f\right\|_{C_{n+1}}}
$$

for all $n$, we have that

$$
\frac{\left\|f_{k}-f\right\|_{C_{n}}}{1+\left\|f_{k}-f\right\|_{C_{n}}}<\rho
$$

for all $n$ as well. Hence $\left\|f_{k}-f\right\|_{C_{n}}<\epsilon$ for all $k \geq M$, and since $C$ is contained in all but finitely many $C_{k}$, we have that $\left\|f_{k}-f\right\|_{C}<\epsilon$. Thus $f_{k}$ converges uniformly to $f$ on $C$.

Conversely, suppose $f_{k}$ converges uniformly to $f$ on compact subsets, and let $\epsilon>0$ be given. Choose a positive integer $N$ for which

$$
\sum_{n=N+1}^{\infty} \frac{1}{2^{n}}<\frac{\epsilon}{2}
$$

Now choose $M$ such that

$$
\left\|f_{k}-f\right\|_{C_{N}}<\frac{\epsilon}{2}
$$

for $k \geq M$. Then

$$
\begin{aligned}
d\left(f_{k}, f\right) & =\sum_{n=1}^{N} \frac{1}{2^{n}} \frac{\left\|f_{k}-f\right\|_{C_{n}}}{1+\left\|f_{k}-f\right\|_{C_{n}}}+\sum_{n=N+1}^{\infty} \frac{1}{2^{n}} \frac{\left\|f_{k}-f\right\|_{C_{n}}}{1+\left\|f_{k}-f\right\|_{C_{n}}} \\
& <\sum_{n=1}^{N} \frac{1}{2^{n}} \frac{\epsilon}{2}+\sum_{n=N+1}^{\infty} \frac{1}{2^{n}} \\
& <\frac{\epsilon}{2}+\frac{\epsilon}{2}=\epsilon
\end{aligned}
$$

Hence $d\left(f_{k}, f\right) \rightarrow 0$.

For the rest of this chapter, let $d$ denote a metric generating the topology of compact convergence on $X$. With a metric on $X$ at hand, we turn our attention to the completeness of $X$. We will 
use the following lemma to show that $X$ is complete.

Lemma 3.3. A sequence $\left\{f_{k}\right\}$ in $X$ converges pointwise to $f$ if and only if $\left\{f_{k}\right\}$ converges to $f$ uniformly on compact subsets of $\mathbb{D}$.

Proof. Certainly uniform convergence on compact subsets implies pointwise convergence. So suppose the sequence $\left\{f_{k}\right\}$ in $X$ converges pointwise to $f$, and let $\epsilon>0$ be given. Let $C \subset \mathbb{D}$ be compact, and let $F \subset \mathbb{D}$ be a compact disk containing $C$ such that the boundary of $F$ is a positive distance $\rho>0$ from $C$. By the Cauchy integral formula, we have that

$$
f_{k}(z)=\frac{1}{2 \pi i} \int_{\partial F} \frac{f_{k}(w)}{w-z} d w \text { for } z \in F .
$$

Fix a point $z$ in $F$. Since $\left\{f_{k}\right\}$ converges pointwise to $f$, and

$$
\left\|\frac{f_{k}(w)}{w-z}\right\|_{\partial F} \leq \frac{1}{\operatorname{dist}(z, \partial F)},
$$

we compute that

$$
\begin{aligned}
f(z) & =\lim _{k \rightarrow \infty} f_{k}(z) \\
& =\lim _{k \rightarrow \infty} \frac{1}{2 \pi i} \int_{\partial F} \frac{f_{k}(w)}{w-z} d w \\
& =\frac{1}{2 \pi i} \int_{\partial F} \frac{f(w)}{w-z} d w,
\end{aligned}
$$

where the last equality follows from the dominated convergence theorem.

By the dominated convergence theorem, we may choose a positive integer $K$ such that

$$
\int_{\partial F}\left|f_{k}(w)-f(w)\right| d w<2 \pi \rho \epsilon
$$


for all $k \geq K$. Then

$$
\begin{aligned}
\left\|f_{k}-f\right\|_{C} & =\sup _{z \in C}\left|\frac{1}{2 \pi i} \int_{\partial F} \frac{f_{k}(w)-f(w)}{w-z} d w\right| \\
& \leq \sup _{z \in C} \frac{1}{2 \pi} \int_{\partial F} \frac{\left|f_{k}(w)-f(w)\right|}{|w-z|} d w \\
& \leq \sup _{z \in C} \frac{1}{2 \pi \operatorname{dist}(z, \partial F)} \int_{\partial F}\left|f_{k}(w)-f(w)\right| d w \\
& \leq \frac{1}{2 \pi \rho} \cdot 2 \pi \rho \epsilon \\
& =\epsilon .
\end{aligned}
$$

Thus $\left\{f_{k}\right\}$ converges uniformly to $f$ on $C$.

One way we can utilize the preceding lemma is to show that $X$ is a complete metric space.

Proposition 3.4. The metric space $(X, d)$ is complete.

Proof. Suppose $\left\{f_{k}\right\}$ is a Cauchy sequence in $X$. Let $z \in \mathbb{D}$. Then the sequence of complex numbers $\left\{f_{k}(z)\right\}$ is Cauchy, so it converges, say to $f(z)$. Since $f_{k} \rightarrow f$ pointwise, $\left|f_{k}(z)\right| \rightarrow|f(z)|$ for each $z$ in $\mathbb{D}$. Hence $|f(z)| \leq 1$ for all $z$ in $\mathbb{D}$. Fix a point $z$ in $\mathbb{D}$ and let $F$ be a compact disk inside of $\mathbb{D}$ containing $z$. We will show that $f(z)$ is differentiable at $z$.

As shown in the proof of Lemma 3.3 .

$$
f(z)=\frac{1}{2 \pi i} \int_{\partial F} \frac{f(w)}{w-z} d w
$$

for all $z$ in $F$. The difference quotient approximating $f^{\prime}(z)$ is given by

$$
\frac{f(z+\Delta z)-f(z)}{\Delta z}=\frac{1}{\Delta z} \frac{1}{2 \pi i} \int_{\partial F}\left(\frac{f(w)}{w-(z+\Delta z)}-\frac{f(w)}{w-z}\right) d w
$$




$$
\begin{aligned}
& =\lim _{\Delta z \rightarrow 0} \frac{1}{\Delta z} \frac{1}{2 \pi i} \int_{\partial F} \frac{f(w)[w-z]-f(w)[w-(z+\Delta z)]}{(w-(z+\Delta z))(w-z)} d w \\
& =\frac{1}{2 \pi i} \int_{\partial F} \frac{f(w)}{(w-(z+\Delta z))(w-z)} d w
\end{aligned}
$$

As $\Delta z$ tends to 0 , the integrand converges uniformly to $\frac{f(w)}{(w-z)^{2}}$. Therefore

$$
\begin{aligned}
f^{\prime}(z) & =\lim _{\Delta z \rightarrow 0} \frac{1}{2 \pi i} \int_{\partial F} \frac{f(w)}{(w-(z+\Delta z))(w-z)} d w \\
& =\frac{1}{2 \pi i} \int_{\partial F} \frac{f(w)}{(w-z)^{2}} d w
\end{aligned}
$$

exists. Thus $f$ is in $X$.

\subsection{Blaschke Products}

Our focus in this section is on the semigroup $X=\overline{\operatorname{Ball}}\left(H^{\infty}(\mathbb{D})\right)=\left\{f \in H^{\infty}(\mathbb{D}):\|f\|_{\infty} \leq\right.$ $1\}$ and Blaschke products. An automorphism of the unit disk $\phi$ is a function $\phi: \mathbb{D} \rightarrow \mathbb{D}$ which is holomorphic and whose inverse is holomorphic. Any automorphism of the unit disk has the form

$$
\phi(z)=\lambda \frac{z-a}{1-\bar{a} z}
$$

where $|\lambda|=1$ and $a$ is a point in $\mathbb{D}$.

A finite Blaschke product $b$ is a finite product of automorphisms of $\mathbb{D}$; that is,

$$
b(z)=\lambda \cdot \prod_{j=1}^{k} \frac{z-a_{j}}{1-\overline{a_{j}} z}, \quad \text { where }\left|a_{j}\right|<1 \text { and }|\lambda|=1 .
$$

Note that $b \in X$. A Blaschke product is a convergent product of finite Blaschke products. A finite Blaschke product $b$ has the following properties:

(i) $b$ is continuous on $\partial \mathbb{D}$. 
(ii) $|b|=1$ on $\partial \mathbb{D}$.

(iii) $b$ has finitely many zeros in $\mathbb{D}$.

In fact, these properties characterize $b$ up to a constant factor of unit modulus. Indeed, suppose $f$ is holomorphic on $\mathbb{D}$ and has properties (i), (ii) and (iii), and let $b$ be the finite Blaschke product formed by the zeros of $f$. Then both $f / b$ and $b / f$ are holomorphic on $\mathbb{D}$ and continuous on $\partial \mathbb{D}$. Further, $|f / b| \leq 1$ and $|b / f| \leq 1$ on the unit circle, so by the maximum modulus principle $f / b$ is a constant of modulus one. We define the degree of $b$ to be its number of zeros.

To apply Corollary 2.2, we need a suitable dense set of $X$ to work with. It turns out that the dense set we will use is a particular collection of Blaschke products. We provide here a proof due to Carathéodory (see [9]) to show that the set of all finite Blaschke products is dense in $X$.

Proposition 3.5. If $f \in X$, then there is a sequence $\left\{b_{k}\right\}$ of finite Blaschke products that converges to $f$ pointwise on $\mathbb{D}$.

Proof. Let $f(z)=\sum_{n=0}^{\infty} a_{n} z^{n}$. For each $n$ we shall find, by induction, a Blaschke product $b_{n}$ of degree at most $n$ whose first $n$ coefficients match those of $f$. This will prove the theorem since the partial sums of $f$ converge uniformly on compact subsets to $f$.

Proceed by induction on $n$. For $n=0$, if $\left|a_{0}\right|=1$ take $b_{0}=a_{0}$. If $\left|a_{0}\right|<1$, then let

$$
b_{0}(z)=\frac{z+a_{0}}{z+\overline{a_{0}} z}
$$

so that $b_{0}(0)=a_{0}$.

Now suppose that for each function in $X$ we have constructed $b_{n-1}(z)$. Let

$$
g(z)=\frac{1}{z} \frac{f(z)-f(0)}{1-\overline{f(0)} f(z)}
$$

so that

$$
f(z)=\frac{z g(z)+f(0)}{1+\overline{f(0)} z g(z)}
$$


Furthermore, since $g$ is holomorphic, we inductively assume there is a Blaschke product $b_{n-1}$ of degree at most $n-1$ such that $g-b_{n-1}$ has $n-1$ zeros at 0 . Then $z g(z)-z b_{n-1}(z)$ has $n$ zeros at 0 . Take

$$
b_{n}(z)=\frac{z b_{n-1}(z)+f(0)}{1+\overline{f(0)} z b_{n-1}(z)} .
$$

Then $b_{n}$ is a finite Blaschke product, and the degree of $b_{n}$ is at most $n$. We compute that

$$
\begin{aligned}
f(z)-b_{n}(z) & =\frac{z g(z)+f(0)}{1+\overline{f(0)} z g(z)}-\frac{z B_{n-1}(z)+f(0)}{1+\overline{f(0)} z B_{n-1}(z)} \\
& =\frac{\left(1-|f(0)|^{2}\right) z\left(g(z)-B_{n-1}(z)\right)}{(1+\overline{f(0)} z g(z))\left(1+\overline{f(0)} z B_{n-1}(z)\right)},
\end{aligned}
$$

which shows that $f-b_{n}$ has a zero of order $n$ at 0 , so that the first $n$ coefficients of $b_{n}$ match those of $f$.

Combining Lemma 3.3 with Proposition 3.5, we see that the collection of all finite Blaschke products forms a dense set in $X$. Hence the countable collection of Blaschke products of the form

$$
B(z)=e^{i \theta} \prod_{j=1}^{n} \frac{z-a_{j}}{1-\overline{a_{j}} z}
$$

where $\theta$ is a rational number and the zeros $a_{n}$ come from a countable dense subset in $\mathbb{D}$ is also a dense set in $X$, which shows that $X$ is separable.

We now move on to prove Heins' result using our Corollary 2.2. The first example of a universal Blaschke product in $X$ was given by Heins in [15] and later in a more general form by Gorkin and Mortini in [12]. Before proving their result, we state four elementary lemmas. The first is a several-variable result which follows from the maximum modulus principle (see [10, Lemma 1]).

Lemma 3.6. If a sequence of holomorphic functions on a domain $\Omega$ in $\mathbb{C}^{N}$ is bounded by 1 , and converges at some point in $\Omega$ to a value of norm 1, then the sequence converges to this value uniformly on compact subsets of $\Omega$.

The previous lemma yields the following several-variable result which is used for the one- 
variable case in the present section, as well as the analogous several-variable case in the ensuing sections. This result provides an important tool in applying Corollary 2.2 .

Lemma 3.7. Let $\left\{\phi_{n}\right\}$ be a sequence of automorphisms of the open unit ball $\mathbb{B}_{N}=\left\{z \in \mathbb{C}^{N}\right.$ : $\|z\|<1\}$ of $\mathbb{C}^{N}$. If $\left\|\phi_{n}(0)\right\| \rightarrow 1$, then there is a subsequence $\left\{\phi_{n_{k}}^{-1}\right\}$ of the autmomorphisms $\left\{\phi_{n}^{-1}\right\}$ for which $\left\|\phi_{n_{k}}^{-1}(0)\right\| \rightarrow 1$.

Proof. By passing to a subsequence if necessary, we may assume there exists $v \in \partial \mathbb{B}_{N}$ for which $\phi_{n}(0) \rightarrow v$. By means of contradiction, suppose there is an $r>0$ such that $\sup _{n}\left\|\phi_{n}^{-1}(0)\right\|<r<1$. Let $K$ be a compact subset of $\mathbb{B}_{N}$ for which $\phi_{n}^{-1}(0) \in K$ for all $n$. By Lemma 3.6, there is a positive integer $M$ such that

$$
\phi_{n}(K) \subset\left\{z \in \mathbb{B}_{N}:\|z-v\|<r\right\}
$$

for all $n \geq M$. But then $0=\phi_{M}\left(\phi_{M}^{-1}(0)\right) \in\left\{z \in \mathbb{B}_{N}:\|z-v\|<r\right\}$, a contradiction. Thus $\sup _{n}\left\|\phi_{n}^{-1}(0)\right\|=1$, and the proof is complete.

The next interpolation result is well known and is useful for us to construct a universal Blaschke product (see [10, Lemma 4]). We then use it to establish a suitable dense set of Blaschke products on which Corollary 2.2 is applied. We state it without proof.

Lemma 3.8. Given complex numbers $b, c \in \mathbb{D}$ and $\alpha_{1}, \alpha_{2}, \eta_{1}, \eta_{2} \in \partial \mathbb{D}$, there exists a finite Blaschke product $\psi$ which satisfies

$$
\psi(b)=c, \quad \psi\left(\alpha_{1}\right)=\eta_{1}, \quad \text { and } \quad \psi\left(\alpha_{2}\right)=\eta_{2},
$$

where if $\alpha_{1}=\alpha_{2}$, then $\eta_{1}=\eta_{2}$.

Equipped with this interpolation result, we now establish the density of certain Blaschke products, which provide a nice dense set in applying Corollary 2.2 . 
Lemma 3.9. For any $v, w \in \partial \mathbb{D}$, the collection of Blaschke products $b$ satisfying $b(v)=1=b(w)$ is dense in $X$.

Proof. By Lemma 3.5, the set of finite Blaschke products is dense in $X$. Let $p$ be a finite Blaschke product. For any positive integer $j$, by Lemma 3.8 there is a finite Blaschke product $\psi_{j}$ such that

$$
\psi_{j}(0)=1-2^{-j}, \quad \psi_{j}(v)=\frac{1}{p(v)}, \quad \psi_{j}(w)=\frac{1}{p(w)}
$$

Consider the finite Blaschke product $b_{j}=\psi_{j} p$. We compute that $b_{j}(v)=1=b_{j}(w)$, and by Lemma 3.6. $b_{j} \rightarrow p$ as $j \rightarrow \infty$. Hence the collection of Blaschke products $b$ which satisfy $b(v)=1=b(w)$ is dense in $X$.

Using the above lemmas, we are now ready to prove the following well known result of Heins [15] and Gorkin and Mortini [12] as an easy application of Corollary 2.2. A more general result is provided in Theorem 3.13 below.

Theorem 3.10. Let $X$ be the topological semigroup $\overline{\mathrm{Ball}}\left(H^{\infty}(\mathbb{D})\right)$ with the topology of comapct convergence. Suppose $\left\{\phi_{n}\right\}$ is a sequence of automorphisms of $\mathbb{D}$. Then there exists a Blaschke product $B$ such that the sequence $\left\{B \circ \phi_{n}\right\}$ is dense in $X$ if and only if $\lim \sup _{n \rightarrow \infty}\left|\phi_{n}(0)\right|=1$.

Proof. First, suppose that $\limsup _{n \rightarrow \infty}\left|\phi_{n}(0)\right|=1$. Passing to a subsequence if necessary, by Lemma 3.6 and Lemma 3.7, we may assume there exist $v, w \in \partial \mathbb{D}$ such that $\phi_{n}(0) \rightarrow v$ and $\phi_{n}^{-1}(0) \rightarrow w$. Using the notation in Corollary 2.2, we define $T_{n}: X \rightarrow X$ by $T_{n} f=f \circ \phi_{n}$ and set $A_{n}=T_{n}^{-1}$. Let $\mathcal{D}_{1}$ be the collection of Blaschke products $b$ such that $b(v)=1=b(w)$, which is dense in $X$ by Lemma 3.9, and take $\mathcal{D}_{2}=\mathcal{D}_{1}$ in Corollary 2.2.

We now check the three hypotheses of Corollary 2.2. Let $b \in \mathcal{D}_{1}$. Since $b(v)=1=b(w)$, we have that $b\left(\phi_{n}(0)\right) \rightarrow b(v)=1$. It follows from Lemma 3.6 that $T_{n} b=b \circ \phi_{n} \rightarrow b(v)=1$, and similarly that $A_{n} b=b \circ \phi_{n}^{-1} \rightarrow b(w)=1$. Lastly, $T_{n} A_{n} b=b$, so the three hypotheses are satisfied 
and there exists a universal element of the form

$$
B=\prod_{j=1}^{\infty} b_{j}
$$

where each $b_{j}$ belongs to $\mathcal{D}_{1}$. Since $B$ is the convergent product of finite Blaschke products, $B$ is a Blaschke product.

For the converse, suppose there is a Blaschke product $B$ for which the sequence $\left\{B \circ \phi_{n}\right\}$ is dense in $X$. Since $B$ is universal, there is a subsequence $\left\{\phi_{n_{k}}\right\}$ for which $B \circ \phi_{n_{k}} \rightarrow 1$. We proceed by contradiction. Suppose that $\lim \sup _{n \rightarrow \infty}\left|\phi_{n}(0)\right| \neq 1$. Then $\left\{\phi_{n_{k}}\right\}$ has a further subsequence, still denoted by $\left\{\phi_{n_{k}}\right\}$, such that $\left\{\phi_{n_{k}}(0)\right\}$ has an accumulation point $a$ in $\mathbb{D}$, which implies that $B(a)=1$. Then $B(z)=1$ for all $z$ in $\mathbb{D}$ by the maximum modulus principle. Since this contradicts the assumption that the set $\left\{B \circ \phi_{n}\right\}$ is dense, the proof is complete.

We now move on to prove a more general form of Theorem 3.10 using Corollary 2.2, where the automorphisms of $\mathbb{D}$ in Theorem 3.10 are replaced with holomorphic self-maps of $\mathbb{D}$, which by definition are sujective holomorphic functions from $\mathbb{D}$ to $\mathbb{D}$. Before proving this generalization we state two lemmas which we will use in the present section as well as the next section. Recall that $\mathcal{N}$ is the semigroup of all zero-free holomorphic functions in $X$ together with the 0 function. The first lemma is a necessary condition for the universality of composition operators which was provided in [4, Section 2.4].

Lemma 3.11. Suppose $\left\{\phi_{n}\right\}$ is a sequence of holomorphic self maps of the open unit disk. If there exists an $f \in X$ such that the closure of $\left\{f \circ \phi_{n}\right\}$ contains $\mathcal{N}$, then

$$
\limsup _{n \rightarrow \infty} \frac{\left|\phi_{n}^{\prime}(0)\right|}{1-\left|\phi_{n}(0)\right|^{2}}=1
$$

In the case when the $\phi_{n}$ are automorphisms, the mappings $A_{n}$ we need to apply Corollary 2.2 are just the composition with $\phi_{n}^{-1}$. However, a holomorphic self-map of $\mathbb{D}$ may not be invertible. Nevertheless, we can use the previous lemma to construct the mappings that we need for Corollary 
2.2

Lemma 3.12. Suppose $\left\{\phi_{n}\right\}$ is a sequence of holomorphic self maps of the open unit disk such that $\left|\phi_{n}(0)\right| \rightarrow 1$. If there exists an $f \in X$ such that the closure of $\left\{f \circ \phi_{n}\right\}$ contains $\mathcal{N}$, then $\left\{\phi_{n}\right\}$ has a subsequence $\left\{\phi_{n_{k}}\right\}$ for which there is a sequence of Blaschke products $\left\{G_{k}\right\}$ with $\left|G_{k}(0)\right| \rightarrow 1$ and $G_{k} \circ \phi_{n_{k}}(z) \rightarrow z$ for all $z \in \mathbb{D}$.

Proof. As a consequence of Lemma 3.11, by passing to a subsequence if necessary, we may assume that

$$
\lim _{n \rightarrow \infty} \frac{\left|\phi_{n}^{\prime}(0)\right|}{1-\left|\phi_{n}(0)\right|^{2}}=1
$$

Recall that a sequence $\left\{z_{n}\right\}$ of distinct points in $\mathbb{D}$ is called thin if

$$
\lim _{k \rightarrow \infty} \prod_{j \neq k}^{\infty}\left|\frac{z_{j}-z_{k}}{1-\overline{z_{j}} z_{k}}\right|=1
$$

Since every sequence in $\mathbb{D}$ converging to the boundary has a thin subsequence (see, for example, [12, Proposition 1.1]), we may also assume, by passing to a further subsequence of $\left\{\phi_{n}(0)\right\}$ if necessary, that there is a $v \in \partial \mathbb{D}$ such that

$$
\phi_{n}(0) \rightarrow v
$$

and $\left\{\phi_{n}(0)\right\}$ is a thin sequence.

Now let $B_{m}$ denote the interpolating Blaschke product corresponding to the thin sequence $\left\{\phi_{n}(0): n \geq m\right\}$. That is, let

$$
B_{m}(z)=\prod_{j=m}^{\infty} \frac{\overline{\phi_{j}(0)}}{\left|\phi_{j}(0)\right|} \frac{\phi_{j}(0)-z}{1-\overline{\phi_{j}(0)} z}
$$

Note that $B_{m}(0)=\prod_{j=m}^{\infty}\left|\phi_{j}(0)\right| \rightarrow 1$ as $m \rightarrow \infty$. A straightforward calculation shows that, for any 
$n \geq m$,

$$
\left(1-\left|\phi_{n}(0)\right|^{2}\right)\left|B_{m}^{\prime}\left(\phi_{n}(0)\right)\right|=\prod_{j \geq m, j \neq n}^{\infty}\left|\frac{\phi_{j}(0)-\phi_{n}(0)}{1-\overline{\phi_{j}(0)} \phi_{n}(0)}\right| .
$$

Hence the assumption that $\left\{\phi_{n}(0): n \geq m\right\}$ be thin means that

$$
\lim _{n \rightarrow \infty}\left(1-\left|\phi_{n}(0)\right|^{2}\right)\left|B_{m}^{\prime}\left(\phi_{n}(0)\right)\right|=1
$$

Computing the derivative of $B_{m} \circ \phi_{n}$ at 0 by the chain rule, and using equation 3.3.1, we obtain that

$$
\left|\left(B_{m} \circ \phi_{n}\right)^{\prime}(0)\right|=\left|B_{m}^{\prime}\left(\phi_{n}(0)\right)\right|\left(1-\left|\phi_{n}(0)\right|^{2}\right) \frac{\left|\phi_{n}^{\prime}(0)\right|}{\left(1-\left|\phi_{n}(0)\right|^{2}\right)} \rightarrow 1 \text {, as } n \rightarrow \infty .
$$

So for each $m \geq 1$, we may choose a positive integer $n_{m} \geq m$ such that

$$
||\left(B_{m} \circ \phi_{n_{m}}\right)^{\prime}(0)|-1|<2^{-m}
$$

Since the collection $\left\{B_{1} \circ \phi_{n_{1}}, B_{2} \circ \phi_{n_{2}}, B_{3} \circ \phi_{n_{3}}, \ldots\right\}$ is a bounded family of holomorphic functions, a normal families argument implies there is a subsequence, still denoted by $\left\{B_{k} \circ \phi_{n_{k}}\right\}_{k=1}^{\infty}$, and a function $g \in X$ such that $B_{k} \circ \phi_{n_{k}} \rightarrow g$. Since $n_{m}$ is chosen so that $n_{m} \geq m$, we have $\left(B_{k} \circ \phi_{n_{k}}\right)(0)=0$ for all $k$ by (3.3.3). Furthermore, $\left|\left(B_{k} \circ \phi_{n_{k}}\right)^{\prime}(0)\right| \rightarrow 1$ by (3.3.4), so we have $g(0)=0$ and $\left|g^{\prime}(0)\right|=1$. Schwarz's lemma now implies that $g(z)=\lambda z$ for some $|\lambda|=1$. Writing $G_{k}=\bar{\lambda} B_{k}$, we see that $G_{k} \circ \phi_{n_{k}}(z) \rightarrow z$ and that $G_{k}(0) \rightarrow \bar{\lambda}$ as $k \rightarrow \infty$.

We are now ready to state and prove a more general form of Theorem 3.10 which generalizes the automorphisms $\phi_{n}$ to holomorphic self-maps. This result was first obtained by Bayart, Gorkin, Grivaux and Mortini [4]. We borrow some of their techniques to expand their result here as a direct application of Corollary 2.2 together with Lemma 3.12

Theorem 3.13. Let $X$ be the topological semigroup $\overline{\mathrm{Ball}}\left(H^{\infty}(\mathbb{D})\right)$ with the topology of compact convergence. Suppose $\left\{\phi_{n}\right\}$ is a sequence of holomorphic self maps of the open unit disk $\mathbb{D}$ such that $\left|\phi_{n}(0)\right| \rightarrow 1$. The following are equivalent: 
(a) there exists $f \in X$ for which the sequence $\left\{f \circ \phi_{n}\right\}$ is dense in $X$;

(b) there exists a sequence of positive integers $\left\{n_{k}\right\}$ and Blaschke products $\left\{G_{k}\right\}$ of $\mathbb{D}$ such that $\left|G_{k}(0)\right| \rightarrow 1$ and $G_{k} \circ \phi_{n_{k}}(z) \rightarrow z$ for all $z \in \mathbb{D} ;$

(c) there exists a Blaschke product $B \in X$ for which the sequence $\left\{B \circ \phi_{n}\right\}$ is dense in $X$.

Proof. It is trivial that statement (c) implies statement (a). We first prove that statement (a) implies statement (b). Since $\mathcal{N} \subset X$, by statement (a) we have that $\left\{f \circ \phi_{n}\right\}$ is also dense in $\mathcal{N}$. Hence statement (b) follows from Lemma 3.12 .

We now proceed to show that statement (b) implies statement (c). By passing to a further subsequence of $\left\{\phi_{n_{k}}\right\}$ and of $\left\{G_{k}\right\}$ if necessary, still denoted by $\left\{\phi_{n_{k}}\right\}$ and $\left\{G_{k}\right\}$, we may assume that there are $v, w \in \partial \mathbb{D}$ such that $\phi_{n_{k}}(0) \rightarrow v$ and $G_{k}(0) \rightarrow w$. Using the notation in Corollary 2.2. we define $T_{k}: X \rightarrow X$ by $T_{k} f=f \circ \phi_{n_{k}}$, and $A_{k}: X \rightarrow X$ by $A_{k} f=f \circ G_{k}$. Take both $\mathcal{D}_{1}$ and $\mathcal{D}_{2}$ in Corollary 2.2 to be the collection of Blaschke products $b$ such that $b(v)=1=b(w)$. Then $\mathcal{D}_{1}$ is a dense subset of $X$ by Lemma 3.9, and $T_{k}$ and $A_{k}$ are continuous homomorphisms on $X$.

We now check that $T_{k}$ and $A_{k}$ satisfy the hypotheses of Corollary 2.2. Let $b \in \mathcal{D}_{1}$. Since $\phi_{n_{k}}(0) \rightarrow v$, Lemma 3.6 implies that $\phi_{n_{k}} \rightarrow v$ uniformly on compact subsets of $\mathbb{D}$. Thus $T_{k} b=$ $b \circ \phi_{n_{k}} \rightarrow b(v)=1$. Similarly, since $G_{k}(0) \rightarrow w$, we have that $G_{k} \rightarrow w$ by Lemma 3.6 . and we compute that $A_{k} b=b \circ G_{k} \rightarrow b(w)=1$. Lastly, since $G_{k} \circ \phi_{n_{k}} \rightarrow z$, we see that $T_{k} A_{k} b=b \circ G_{k} \circ \phi_{n_{k}} \rightarrow b$. Hence the three hypotheses in Corollary 2.2 are satisfied, so $\left\{T_{k}\right\}$ is universal and there exists a universal element of the form

$$
B=\prod_{j=1}^{\infty} b_{j}
$$

where each $b_{j}$ belongs to $\mathcal{D}_{1}$. Thus $B$ is a Blaschke product, and the proof of statement (c) is complete.

There are two types of inner functions on $\mathbb{D}$, namely the Blaschke products and the singular 
inner functions. In this section, we have dealt with the universality of Blaschke products for composition operators. In the next section, we deal with universal singular inner functions for the same sequence of composition operators as in Theorem 3.13 .

\subsection{Singular Inner Functions}

In this section, we continue to consider the topological semigroup $X=\overline{\operatorname{Ball}}\left(H^{\infty}(\mathbb{D})\right)$ with the topology of compact convergence. There is an analogous result to Theorem 3.13 for the set of all zero-free holomorphic functions in $X$. The universal function in this case is a singular inner function, which by definition is a function of the form

$$
S_{\mu}(z)=e^{i \theta} \exp \left(-\int_{|\zeta|=1} \frac{\zeta+z}{\zeta-z} d \mu(\zeta)\right)
$$

where $\mu$ is a singular, positive Borel measure on $\partial \mathbb{D}$, and $\theta \in \mathbb{R}$.

Recall that $\mathcal{N}$ is the semigroup of all zero-free holomorphic functions in $X$ together with the 0 function. Before proving the main result of this section we state two lemmas, the first of which can be found in [13, Corollary 1.3].

Lemma 3.14. The set $\mathcal{D}$ of discrete singular inner functions of the form

$$
e^{i q} \prod_{k=0}^{n-1} \exp \left(-\epsilon_{k, n} \frac{e^{2 \pi i k / n}+z}{e^{2 \pi i k / n}-z}\right)
$$

where $q \in \mathbb{Q}, n \in \mathbb{N}, \epsilon_{k, n} \in \mathbb{Q}$ and $\epsilon_{k, n}>0$, is dense in $\mathcal{N}$.

The set of discrete singular inner functions of the above form plays the role of the finite Blaschke products from the previous section. Following the same line of thought, the next lemma is an analogue of Lemmas 3.8 and 3.9 for $\mathcal{N}$. In fact, we will use the interpolation result in Lemma 3.8 to prove our lemma. It provides a nice dense set of singular inner functions in $\mathcal{N}$ that allows us easily to apply Corollary 2.2 .

Lemma 3.15. Given $\epsilon>0$ and $\alpha_{1}, \alpha_{2}, \eta_{1}, \eta_{2} \in \partial \mathbb{D}$, there exists a singular inner function $\sigma$ in $\mathcal{N}$ 
such that

$$
\sigma(0)>1-\epsilon, \quad \sigma\left(\alpha_{1}\right)=\eta_{1}, \quad \text { and } \quad \sigma\left(\alpha_{2}\right)=\eta_{2} .
$$

where if $\alpha_{1}=\alpha_{2}$, then $\eta_{1}=\eta_{2}$. Consequently, the set of singular inner functions $s$ such that $s\left(\alpha_{1}\right)=1=s\left(\alpha_{2}\right)$ is dense in $\mathcal{N}$.

Proof. Let $\delta>0$ be such that $e^{-\delta}>1-\epsilon$ and define $H(z)=\exp \left(-\delta \frac{1+z}{1-z}\right)$. Then $H$ is a singular inner function with $H(0)>1-\epsilon$. By Lemma 3.8 , there exists a finite Blaschke product $B$ such that

$$
B(0)=0, B\left(\alpha_{1}\right) \text { is in the set } H^{-1}\left\{\eta_{1}\right\} \text {, and } B\left(\alpha_{2}\right) \text { is in the set } H^{-1}\left\{\eta_{2}\right\} \text {. }
$$

Then $\sigma=H \circ B$ is a singular inner function which satisfies 3.4.1.

It remains to show the set of singular inner functions $s$ such that $s\left(\alpha_{1}\right)=1=s\left(\alpha_{2}\right)$ is dense in $\mathcal{N}$. For that, we observe that by Lemma 3.14, the set of singular inner functions which are analytic at $\alpha_{1}$ and $\alpha_{2}$ is dense in $\mathcal{N}$. Let $S$ be any such function. Since $\left|S\left(\alpha_{1}\right)\right|=1=\left|S\left(\alpha_{2}\right)\right|$, we may choose for each positive integer $j$ a singular inner function $\sigma_{j}$ such that

$$
\sigma_{j}(0)>1-2^{-j}, \quad \sigma_{j}\left(\alpha_{1}\right)=\frac{1}{S\left(\alpha_{1}\right)} \text { and } \sigma_{j}\left(\alpha_{2}\right)=\frac{1}{S\left(\alpha_{2}\right)}
$$

Now consider the singular inner function $s_{j}=\sigma_{j} \cdot S$. We compute that $s_{j}\left(\alpha_{1}\right)=1=s_{j}\left(\alpha_{2}\right)$, and by Lemma 3.6, we have $s_{j} \rightarrow S$ as $j \rightarrow \infty$. The proof is complete.

Using the density of this special class of singular inner functions in $\mathcal{N}$, we now use Corollary 2.2 to provide an easy proof of the following theorem, which was first obtained by Gorkin and Mortini [13] and Bayart, Gorkin, Grivaux and Mortini [4]. The proof is analogous to that of Theorem 3.13 ,

Theorem 3.16. Let $\mathcal{N}$ be the topolocial semigroup $\mathcal{N}=\left\{f \in \overline{\mathrm{Ball}}\left(H^{\infty}(\mathbb{D})\right): f(z) \neq 0\right.$ for all $z$ in $\mathbb{D}\} \cup\{0\}$ with the topology of compact convergence. Suppose $\left\{\phi_{n}\right\}$ is a sequence of holomorphic self maps of the open unit disk such that $\left|\phi_{n}(0)\right| \rightarrow 1$. The following are equivalent: 
(a) there exists $f \in \mathcal{N}$ for which the sequence $\left\{f \circ \phi_{n}\right\}$ is dense in $\mathcal{N}$;

(b) there exists a sequence of positive integers $\left\{n_{k}\right\}$ and Blaschke products $\left\{G_{k}\right\}$ of $\mathbb{D}$ such that $\left|G_{k}(0)\right| \rightarrow 1$ and $G_{k} \circ \phi_{n_{k}}(z) \rightarrow z$ for all $z \in \mathbb{D} ;$

(c) there exists a singular inner function $S \in \mathcal{N}$ for which the sequence $\left\{S \circ \phi_{n}\right\}$ is dense in $\mathcal{N}$.

Proof. It is trivial that statement (c) implies statement (a). Furthermore, Lemma 3.12 yields that statement (a) implies statement (b).

It remains to show that statement (b) implies statement (c). By passing to a further subsequence of $\left\{\phi_{n_{k}}\right\}$ and of $\left\{G_{k}\right\}$ if necessary, still denoted by $\left\{\phi_{n_{k}}\right\}$ and $\left\{G_{k}\right\}$, we may assume that there are $v, w \in \partial \mathbb{D}$ such that $\phi_{n_{k}}(0) \rightarrow v$ and $G_{k}(0) \rightarrow w$. Using the notation in Corollary 2.2, we define $T_{k}: X \rightarrow X$ by $T_{k} f=f \circ \phi_{n_{k}}$, and $A_{k}: X \rightarrow X$ by $A_{k} f=f \circ G_{k}$. Take both $\mathcal{D}_{1}$ and $\mathcal{D}_{2}$ in Corollary 2.2 to be the collection of singular inner functions $s$ such that $s(v)=1=s(w)$, which is dense in $\mathcal{N}$ by Lemma 3.15 .

We now check that $T_{k}$ and $A_{k}$ satisfy the hypotheses of Corollary 2.2. Let $s \in \mathcal{D}_{1}$. Since $\phi_{n_{k}}(0) \rightarrow v$, Lemma 3.6 implies that $\phi_{n_{k}} \rightarrow v$ uniformly on compact subsets of $\mathbb{D}$. Thus $T_{k} s=$ $s \circ \phi_{n_{k}} \rightarrow s(v)=1$. Similarly, since $G_{k}(0) \rightarrow w$, we have that $G_{k} \rightarrow w$ by Lemma 3.6 . and we compute that $A_{k} s=s \circ G_{k} \rightarrow s(w)=1$. Lastly, since $G_{k} \circ \phi_{n_{k}} \rightarrow z$, we see that $T_{k} A_{k} s=s \circ G_{k} \circ \phi_{n_{k}} \rightarrow s$. Hence the three hypotheses in Corollary 2.2 are satisfied, so $\left\{T_{k}\right\}$ is universal and there exists a universal element of the form

$$
S=\prod_{j=1}^{\infty} s_{j}
$$

where each $s_{j}$ belongs to $\mathcal{D}_{1}$. Since the convergent product of singular inner functions is a singular inner function (see [3, Proposition 2]), $S$ is a singular inner function, and the proof of (b) $\Longrightarrow$ (c) is complete.

Since every inner function in $H^{\infty}(\mathbb{D})$ is a product of a Blaschke product and a singular inner function, our discussions above show that each of those two types of inner functions has nice 
universality properties. However, for the case of several complex variables, inner functions are not as simple.

\subsection{Inner Functions in Several Variables}

After we have obtained a few one-variable results, we now turn our attention to several variables. In particular, we prove the analogous result of Theorem 3.10 for the unit ball $\mathbb{B}_{N}=\{z \in$ $\left.\mathbb{C}^{N}:\|z\|<1\right\}$ of $\mathbb{C}^{N}$. Recall that $\mathcal{B}=\overline{\text { Ball }}\left(H^{\infty}\left(\mathbb{B}_{N}\right)\right)=\left\{f \in H^{\infty}\left(\mathbb{B}_{N}\right):\|f\|_{\infty} \leq 1\right\}$. Let $A\left(\overline{\mathbb{B}_{N}}\right)$ be the ball algebra; that is,

$$
A\left(\overline{\mathbb{B}_{N}}\right)=\left\{f \in C\left(\overline{\mathbb{B}_{N}}\right): f \text { holomorphic on } \mathbb{B}_{N}\right\}
$$

We collect three important lemmas for our purpose. The first is a lemma of approximation of functions in $\mathcal{B}$ by functions in $A\left(\overline{\mathbb{B}_{N}}\right)$. It is due to Chee [7].

Lemma 3.17. Let $h \in \mathcal{B}, 0<R<1, \epsilon>0$ and $u \in \partial \mathbb{B}_{N}$. Then there exists $g \in A\left(\overline{\mathbb{B}_{N}}\right)$ such that

$$
\|g\|_{\infty} \leq 1, \quad\|h-g\|_{R \overline{\mathbb{B}_{N}}}<\epsilon, \quad \text { and } g(u)=1 .
$$

A class of holomorphic functions between $A\left(\overline{\mathbb{B}_{N}}\right)$ and $H^{\infty}\left(\mathbb{B}_{N}\right)$ plays an important role in the construction of our inner function, namely the class $M\left(\mathbb{B}_{N}\right)$ of all $f \in H^{\infty}\left(\mathbb{B}_{N}\right)$ each of which has a continuous extension to $\mathbb{B}_{N} \cup \Gamma_{f}$ for some set $\Gamma_{f} \subset \partial \mathbb{B}_{N}$ with $\sigma\left(\Gamma_{f}\right)=1$, where $\sigma$ is the normalized Lebesgue measure on $\partial \mathbb{B}_{N}$. The second lemma we need is the following version of Alexandrov's interpolation theorem [1].

Lemma 3.18. Let $g \in A\left(\overline{\mathbb{B}_{N}}\right)$ such that there exists a non-zero function $U \in A\left(\overline{\mathbb{B}_{N}}\right)$ with $|g|+$ $|U| \leq 1$ on $\overline{\mathbb{B}_{N}}$. If $G \in M\left(\mathbb{B}_{N}\right)$, then there exists an inner function I such that $I-g \in G \cdot H^{\infty}\left(\mathbb{B}_{N}\right)$.

Lastly, using the previous two lemmas, we prove an analogue of Lemma 3.9 for $\mathbb{B}_{N}$. The following lemma is the bulk of our work in this section, as it supplies us with nice dense sets of inner functions on which to apply Corollary 2.2 . 
Lemma 3.19. Suppose $\left\{v_{n}\right\}$ is a sequence in $\mathbb{B}_{N}$ which converges to $v \in \partial \mathbb{B}_{N}$. Then there is a subsequence $\left\{v_{n_{k}}\right\}$ such that the set of inner functions I satisfying $I\left(v_{n_{k}}\right) \rightarrow 1$ is dense in $\mathcal{B}$.

Proof. Let $d$ denote a metric giving the topology of $\mathcal{B}$. Let $h \in \mathcal{B}$ and $\epsilon>0$ be given. By Lemma 3.17, there is a function $f \in A\left(\overline{\mathbb{B}_{N}}\right)$ such that $\|f\|_{\infty} \leq 1, f(v)=1$ and $d(f, h)<2^{-3} \epsilon$.

Let $P_{v}(z)=\langle z, v\rangle$. Since $\left\{P_{v}\left(v_{n}\right)\right\}$ is a sequence of points in $\mathbb{D}$ converging to 1 , there is a subsequence $\left\{P_{v}\left(v_{n_{k}}\right)\right\}$ which is a Blaschke sequence. For any integer $m \geq 1$, let $B_{v}$ be the Blaschke product whose zeros are 0 and $\left\{P_{v}\left(v_{n_{k}}\right)\right\}$, all of which have order $m$. Then the function $G(z)=B_{v}\left(P_{v}(z)\right)$ belongs to $M\left(\mathbb{B}_{N}\right)$.

Let $\delta>0$ and define

$$
g(z)=(1-\delta) f(z)+\delta\left(\frac{1+P_{v}(z)}{2}\right)^{2}
$$

and

$$
U(z)=\delta\left(\frac{1-P_{v}(z)}{2}\right)^{2}
$$

Then $g(v)=1$ and $U$ does not vanish on $\mathbb{B}_{N}$. Note that

$$
g(z)-f(z)=-\delta f(z)+\delta\left(\frac{1+P_{v}(z)}{2}\right)^{2}
$$

Hence we can choose $\delta>0$ so that $d(g, f)<2^{-3} \epsilon$. We compute that

$$
|g|+|U| \leq(1-\delta)|f|+\frac{\delta}{4}\left(1+P_{v}\right)\left(1+\overline{P_{v}}\right)+\frac{\delta}{4}\left(1-P_{v}\right)\left(1-\overline{P_{v}}\right) \leq 1
$$

Lemma 3.18 now provides an inner function $I$ such that $I-g$ is divisible by $G$. In particular, $m$ can be chosen large enough so that $d(I, g)<2^{-3} \epsilon$ (see [3, Theorem 2]). Since $G\left(v_{n_{k}}\right)=0$ for all $k$, we have that $I\left(v_{n_{k}}\right)-g\left(v_{n_{k}}\right)=0$ for all $k$. Therefore, since $g(v)=1$ and $g$ is continuous at $v$, we have that $I\left(v_{n_{k}}\right)=g\left(v_{n_{k}}\right) \rightarrow 1$ and $d(I, h) \leq d(I, g)+d(g, f)+d(f, h)<\epsilon$, which finishes the proof.

Using the above lemma and a direct application of Corollary 2.2, we now prove Theorem 3.20, 
which is a several-variable analogue of Theorem 3.10 . In doing so, we simplify the proof given by Bayart [2] as well as unify his result with the others from this chapter by putting them all in the semigroup setting of Corollary 2.2 .

Theorem 3.20. Let $\mathcal{B}$ be the topological semigroup $\mathcal{B}=\overline{\mathrm{Ball}}\left(H^{\infty}\left(\mathbb{B}_{N}\right)\right)$ with the topology of compact convergence. Suppose $\left\{\phi_{n}\right\}$ is a sequence of automorphisms of the ball $\mathbb{B}_{N}$. Then $\lim \sup _{n \rightarrow \infty}$ $\left\|\phi_{n}(0)\right\|=1$ if and only if there is a sequence of integers $\left\{n_{k}\right\}$ such that the set $\mathcal{D}_{1}=\{I \in \mathcal{B}: I$ is inner and $\left.I \circ \phi_{n_{k}}(0) \rightarrow 1\right\}$ is dense in $\mathcal{B}$ and there exists a universal inner function

$$
I=\prod_{j=1}^{\infty} I_{j}
$$

where each $I_{j}$ belongs to $\mathcal{D}_{1}$.

Proof. First, suppose that $\lim \sup _{n \rightarrow \infty}\left\|\phi_{n}(0)\right\|=1$. By passing to a subsequence if necessary, by Lemma 3.7 we may assume that there are $v, w \in \partial \mathbb{B}_{N}$ such that $\phi_{n}(0) \rightarrow v$ and $\phi_{n}^{-1}(0) \rightarrow w$. Then, by applying Lemma 3.19 twice, there is a sequence of integers $\left\{n_{k}\right\}$ such that the sets $\mathcal{D}_{1}=\left\{I \in \mathcal{B}: I\right.$ is inner and $\left.I \circ \phi_{n_{k}}(0) \rightarrow 1\right\}$ and $\mathcal{D}_{2}=\left\{J \in \mathcal{B}: J\right.$ is inner and $\left.J \circ \phi_{n_{k}}^{-1}(0) \rightarrow 1\right\}$ are both dense in $\mathcal{B}$.

We now apply Corollary 2.2. Following its notations, define $T_{k}: \mathcal{B} \rightarrow \mathcal{B}$ by $T_{k} f=f \circ \phi_{n_{k}}$ and define $A_{k}: \mathcal{B} \rightarrow \mathcal{B}$ by $A_{k} f=T_{k}^{-1} f=f \circ \phi_{n_{k}}^{-1}$. If $I \in \mathcal{D}_{1}$, then $T_{k} I=I \circ \phi_{n_{k}} \rightarrow 1$ by Lemma 3.6. Similary, if $J \in \mathcal{D}_{2}$, then $A_{k} J=J \circ \phi_{n_{k}} \rightarrow 1$. Since $T_{k} A_{k} J=J$, all three conditions of Corollary 2.2 are satisfied. Thus $\left\{T_{k}\right\}$ is universal and there is a universal element of the form

$$
I=\prod_{j=1}^{\infty} I_{j}
$$

where each $I_{j}$ belongs to $\mathcal{D}_{1}$. Since the convergent product of inner functions is an inner function (see [10, Lemma 5]), the proof is complete.

For the converse, we proceed by contradiction. Assume that $\lim _{\sup } \rightarrow \infty\left\|\phi_{n}(0)\right\| \neq 1$. If there is an inner function $I$ for which the sequence $\left\{I \circ \phi_{n}\right\}$ is dense in $\mathcal{B}$, then by our assumption, there is a further subsequence, still denoted by $\left\{\phi_{n_{k}}\right\}$, such that $\left\{\phi_{n_{k}}(0)\right\}$ has an accumulation 
point $a$ in $\mathbb{B}_{N}$. This implies that $I(a)=1$, and that $I(z)=1$ for all $z$ in $\mathbb{B}_{N}$ by the maximum modulus principle. Since this contradicts the assumption that the set $\left\{I \circ \phi_{n}\right\}$ is dense, the proof is complete.

Universal inner functions can also be constructed on more general domains in $\mathbb{C}^{N}$ using the same techniques above, and we refer the reader to [3] for more details. We now turn our attention to a new application of Corollary 2.2 on the ball of an operator algebra. 


\section{CHAPTER 4 UNIVERSALITY ON THE CLOSED UNIT BALL OF THE OPERATOR ALGEBRA OF CLASSICAL BANACH SPACES}

\subsection{Introduction}

Let $Y$ be either $c_{0}$ or $\ell^{p}, 1 \leq p<\infty$, and denote by $\left\{e_{k}: k \geq 1\right\}$ the canonical basis of $Y$. Let $\mathcal{L}(Y, Y)$ be the operator algebra of $Y$, and let $X$ be the closed unit ball of $\mathcal{L}(Y, Y)$. With the usual multiplication of operators, $X$ is a semigroup whose identity element is the identity operator $I$.

Let $U \in X$ be an isometric isomorphism and consider the map $T_{U}$ which conjugates an operator $V$ in $X$ by $U$; that is, $T_{U}: X \rightarrow X$ is given by $T_{U}(V)=U^{-1} V U$. Since

$$
\begin{aligned}
T_{U}(V W) & =U^{-1} V W U \\
& =U^{-1} V U U^{-1} W U \\
& =T_{U}(V) T_{U}(W)
\end{aligned}
$$

$T_{U}$ is a homomorphism of $\mathcal{L}(Y, Y)$ with $T_{U}(I)=I$. Our goal is to use Corollary 2.2 to provide a sufficient condition in Theorem 4.8 for a sequence of such conjugation maps to be universal when $X$ is endowed with the strong operator topology. We first recall the strong operator topology and show that $X$ is metrizable, complete and separable with this topology.

\subsection{The Strong Operator Topology}

The strong operator topology, which we denote by $\tau$, is the weakest topology on $\mathcal{L}(Y, Y)$ such that the evaluation maps

$$
E_{x}: \mathcal{L}(Y, Y) \rightarrow Y
$$

given by $E_{x}(T)=T x$ are continuous for all $x \in Y$. It turns out that a basis for $\tau$ is given by the collection of sets of the form

$$
\left\{T \in \mathcal{L}(Y, Y):\left\|\left(T-T_{0}\right) x_{i}\right\|<\epsilon, i=1, \ldots, n\right\}
$$


where $T_{0} \in \mathcal{L}(Y, Y), x_{i} \in Y$ and $\epsilon>0$ are given. Since we could not find a proof of the equivalence of these two definitions in the literature, our first goal is to prove their equivalence. For now, let $\mathcal{B}^{\prime}$ be the basis above and let $\tau^{\prime}$ be the topology generated by $\mathcal{B}^{\prime}$. We will show $\tau=\tau^{\prime}$. To do so, we recall some important definitions and lemmas.

If $\tau$ and $\tau^{\prime}$ are two topologies on a set $S$, we say $\tau^{\prime}$ is weaker than $\tau$ if $\tau^{\prime} \subset \tau$. The usefulness of bases, for our purposes, is that they offer a tool when trying to compare two topologies given in Lemma 4.2 below. To use Lemma 4.2, we must first recover a basis for $\tau$ from the definition of $\tau$. Recall that a subbasis $\mathcal{C}$ for a topology on $S$ is a collection of subsets of $S$ whose union equals $S$. The topology generated by the subbasis $\mathcal{C}$ is defined to be the collection of all unions of finite intersections of elements of $\mathcal{C}$. In fact, if $\mathcal{C}$ is a subbasis for a topology on a set $S$, a basis $\mathcal{B}$ for the topology generated by $\mathcal{C}$ is given by the collection of all finite intersections of elements of $\mathcal{C}$ (see, for example, [19, page 82]). We now exhibit a subbasis for the topology $\tau$.

Lemma 4.1. The collection of subsets of $\mathcal{L}(Y, Y)$ given by $\mathcal{C}=\left\{E_{x}^{-1}(V): x \in Y, V \subset Y\right.$ is open\} is a subbasis for $\tau$.

Proof. Let $T \in \mathcal{L}(Y, Y)$ be given, and let $x$ be any element in the preimage $T^{-1}(Y)$. Then $T \in E_{x}^{-1}(Y)$, and since $Y$ is open in $Y$, we have shown that $\mathcal{C}$ is a subbasis for some topology on $\mathcal{L}(Y, Y)$. We must show that topology is $\tau$.

By the definition of $\tau$, every member of $\mathcal{C}$ must belong to $\tau$. Then since $\mathcal{C} \subset \tau$, the topology generated by the subbasis $\mathcal{C}$ is necessarily weaker than $\tau$. Since the topology generated by $\mathcal{C}$ is a topology for which the evaluation maps $E_{x}$ are continuous, and $\tau$ is defined to be the weakest topology such that this property holds, we must have that $\tau$ is equal to the topology generated by $\mathcal{C}$.

Let $\mathcal{B}$ be the basis for $\tau$ provided by the subbasis in Lemma 4.1 , that is, let $\mathcal{B}$ be collection of all sets of the form

$$
E_{x_{1}}^{-1}\left(V_{1}\right) \cap \cdots \cap E_{x_{n}}^{-1}\left(V_{n}\right)
$$

where $x_{1}, \ldots, x_{n} \in Y$ and $V_{1}, \ldots, V_{n}$ are open subsets of $Y$. To show $\tau=\tau^{\prime}$, we will employ the 
following fundamental result from topology (see [19, Lemma 13.3]).

Lemma 4.2. Let $\mathcal{A}$ and $\mathcal{A}^{\prime}$ be bases for the topologies $\sigma$ and $\sigma^{\prime}$, respectively, on a set $S$. Then the following are equivalent:

(1) $\sigma^{\prime}$ is weaker than $\sigma$;

(2) For each $T \in S$ and each basis element $A \in \mathcal{A}$ containing $T$, there is a basis element $A^{\prime} \in \mathcal{A}^{\prime}$ such that $T \in A^{\prime} \subset A$.

With the bases $\mathcal{B}$ and $\mathcal{B}^{\prime}$ defined above for the topologies $\tau$ and $\tau^{\prime}$, we now use Lemma 4.2 to show that $\tau=\tau^{\prime}$.

Proposition 4.3. The topologies $\tau$ and $\tau^{\prime}$ on $\mathcal{L}(Y, Y)$ are equal.

Proof. We first show that $\tau^{\prime} \subset \tau$. Let $T \in \mathcal{L}(Y, Y)$ and $B \in \mathcal{B}$ with $T \in B$. We must find $B^{\prime} \in \mathcal{B}^{\prime}$ such that $T \in B^{\prime}$ and $B^{\prime} \subset B$.

By the definition of the basis $\mathcal{B}$, there are elements $x_{1}, \ldots, x_{n}$ in $Y$ and open subsets $V_{1}, \ldots, V_{n}$ in $Y$ such that

$$
B=E_{x_{1}}^{-1}\left(V_{1}\right) \cap \cdots \cap E_{x_{n}}^{-1}\left(V_{n}\right) .
$$

Since $T \in B$, we have that $T x_{i} \in V_{i}$ for each $i=1, \ldots, n$. Since each $V_{i}$ is open, there is some $r_{i}>0$ for which the ball of radius $r_{i}$ centered at $T x_{i}$ is contained in $V_{i}$. Let $\epsilon$ be the smallest such $r_{i}$, and define

$$
B^{\prime}=\left\{T^{\prime}:\left\|\left(T-T^{\prime}\right) x_{i}\right\|<\epsilon, i=1, \ldots, n\right\}
$$

Clearly $T \in B^{\prime}$. If $T^{\prime} \in B^{\prime}$, then $T^{\prime} x_{i}$ is contained inside the ball of radius $r_{i}$ centered at $T x_{i}$ for all $i=1, \ldots, n$. Hence $T^{\prime} x_{i} \in V_{i}$ for each $i$, which shows that $T^{\prime} \in B$. Thus $B^{\prime} \subset B$, and Lemma 4.2 yields that $\tau^{\prime} \subset \tau$.

We now show that $\tau \subset \tau^{\prime}$. Let $T \in \mathcal{L}(Y, Y)$ and $B^{\prime} \in \mathcal{B}^{\prime}$ with $T \in B^{\prime}$. We must find $B \in \mathcal{B}$ such that $T \in B$ and $B \subset B^{\prime}$. 
By the definition of the basis $\mathcal{B}^{\prime}$, there are elements $x_{1}, \ldots, x_{n}$ in $Y$ and $\epsilon>0$ for which

$$
B^{\prime}=\left\{T^{\prime}:\left\|\left(T-T^{\prime}\right) x_{i}\right\|<\epsilon \text { for } i=1, \ldots, n\right\}
$$

Let $V_{i}$ be the open ball of radius $\epsilon$ centered at $T x_{i}$, and define

$$
B=E_{x_{1}}^{-1}\left(V_{1}\right) \cap \cdots \cap E_{x_{n}}^{-1}\left(V_{n}\right)
$$

Clearly $T \in B$. If $T_{0} \in B$, then $T_{0} x_{i} \in V_{i}$ for each $i$. Hence $\left\|\left(T-T_{0}\right) x_{i}\right\|<\epsilon$ for each $i$, which shows that $T_{0} \in B^{\prime}$. Thus $B \subset B^{\prime}$, and Lemma 4.2 yields that $\tau \subset \tau^{\prime}$.

In practice, characterizations of convergence in a topology are easier to work with than the bases defining a topology. We now show that convergence in the strong operator topology is equivalent to pointwise convergence.

Proposition 4.4. For an operator $T \in \mathcal{L}(Y, Y)$, a net of operators $\left\{T_{\alpha}\right\}$ in $\mathcal{L}(Y, Y)$ converges to $T$ in the strong operator topology if and only if $\left\|\left(T_{\alpha}-T\right) x\right\| \rightarrow 0$ for all $x \in Y$.

Proof. Suppose that $\left\|\left(T_{\alpha}-T\right) x\right\| \rightarrow 0$ for all $x \in Y$. Let $V$ be any open set in $\tau$ containing $T$, and let $B$ be a basis element with $T \in B \subset V$. Then there exist $x_{1}, \ldots, x_{k} \in Y$ and $\epsilon>0$ such that $B$ has the form

$$
B=\left\{T^{\prime}:\left\|\left(T^{\prime}-T\right) x_{i}\right\|<\epsilon \text { for } i=1, \ldots, k\right\}
$$

For each $i=1, \ldots, k$, we have that $\left\|\left(T_{\alpha}-T\right) x_{i}\right\| \rightarrow 0$, so we may choose a $\beta$ so that whenver $\alpha \geq \beta$, we have

$$
\left\|\left(T_{\alpha}-T\right) x_{i}\right\|<\epsilon
$$

Thus $T_{\alpha} \in B$ for all $\alpha \geq \beta$, which shows that $T_{\alpha}$ is eventually in $U$ and that $T_{\alpha} \rightarrow T$ in the strong operator topology.

Conversely, suppose that $T_{\alpha} \rightarrow T$ in the strong operator topology. Let $x \in Y$ and $\epsilon>0$ be 
given. Since the set

$$
B=\left\{T^{\prime}:\left\|\left(T^{\prime}-T\right) x\right\|<\epsilon\right\}
$$

is open in $\tau$, there exists some $\beta$ so that when $\alpha \geq \beta$, we have $T_{\alpha} \in B$. Thus whenever $\alpha \geq \beta$, we have $\left\|\left(T_{\alpha}-T\right) x\right\|<\epsilon$, which shows that $\left\|\left(T_{\alpha}-T\right) x\right\| \rightarrow 0$ and completes the proof.

We are now equipped to show that $X$ is metrizable with the strong operator topology.

Proposition 4.5. The set $X$ endowed with the strong operator topology is metrizable, and a metric which gives the strong operator topology is

$$
d\left(T, T_{0}\right)=\sum_{k=1}^{\infty} \frac{1}{2^{k}}\left\|\left(T-T_{0}\right) e_{k}\right\| .
$$

Proof. It is clear that $d$ is a metric. We show that the topology induced by $d$ is equal to $\tau$ by showing that a net of operators $\left\{T_{\alpha}\right\}$ in $\mathcal{L}(Y, Y)$ to $T$ in the strong operator topology if and only if $d\left(T_{\alpha}, T\right) \rightarrow 0$.

Suppose first that $T_{\alpha} \rightarrow T$ in the strong operator topology. Let $\epsilon>0$ be given. Choose a positive integer $N$ so that

$$
\sum_{k=N+1}^{\infty} \frac{2}{2^{k}}<\frac{\epsilon}{2}
$$

For each $i=1, \ldots, N$, Proposition 4.4 implies that $\left\|\left(T_{\alpha}-T\right) x_{i}\right\| \rightarrow 0$. So choose $\beta$ such that whenever $\alpha \geq \beta$, we have that $\left\|\left(T_{\alpha}-T\right) x_{i}\right\|<\epsilon / 2$. Then for $\alpha \geq \beta$,

$$
\begin{aligned}
d\left(T_{\alpha}, T\right) & =\sum_{k=1}^{\infty} \frac{1}{2^{k}}\left\|\left(T_{\alpha}-T\right) e_{k}\right\| \\
& =\sum_{k=1}^{N} \frac{1}{2^{k}}\left\|\left(T_{\alpha}-T\right) e_{k}\right\|+\sum_{k=N+1}^{\infty} \frac{1}{2^{k}}\left\|\left(T_{\alpha}-T\right) e_{k}\right\| \\
& \leq \sum_{k=1}^{N} \frac{1}{2^{k}} \frac{\epsilon}{2}+\sum_{k=N+1}^{\infty} \frac{1}{2^{k}}\left\|T_{\alpha}-T\right\| \cdot\left\|e_{k}\right\| \\
& \leq \frac{\epsilon}{2}+\sum_{k=N+1}^{\infty} \frac{2}{2^{k}}
\end{aligned}
$$




$$
\leq \frac{\epsilon}{2}+\frac{\epsilon}{2}=\epsilon
$$

Hence $d\left(T_{\alpha}, T\right) \rightarrow 0$.

Conversely, suppose that $d\left(T_{\alpha}, T\right) \rightarrow 0$. Let $j$ be any positive integer, and let $\epsilon>0$ be given. Choose $\beta$ such that for $\alpha \geq \beta$, we have

$$
d\left(T_{\alpha}, T\right)<\frac{\epsilon}{2^{j}}
$$

Then

$$
\frac{1}{2^{j}}\left\|\left(T_{\alpha}-T\right) e_{j}\right\|<\frac{\epsilon}{2^{j}}
$$

which implies that $\left\|\left(T_{\alpha}-T\right) e_{j}\right\|<\epsilon$. Hence $\left\|\left(T_{\alpha}-T\right) e_{j}\right\| \rightarrow 0$, and by linearity, $T_{\alpha} \rightarrow T$ pointwise on $\operatorname{span}\left\{e_{1}, e_{2}, \ldots\right\}$. Now let $x \in Y$ and choose $y \in \overline{\operatorname{span}}\left\{e_{1}, e_{2}, \ldots\right\}$ such that $\| x-$ $y \|<\epsilon / 4$. Choose $\beta$ such that $\left\|\left(T_{\alpha}-T\right) y\right\|<\epsilon / 2$ whenever $\alpha \geq \beta$. Then, for any $\alpha \geq \beta$,

$$
\begin{aligned}
\left\|\left(T_{\alpha}-T\right) x\right\| & \leq\left\|\left(T_{\alpha}-T\right)(x-y)\right\|+\left\|\left(T_{\alpha}-T\right) y\right\| \\
& \leq\left\|\left(T_{\alpha}-T\right)\right\| \cdot\|(x-y)\|+\frac{\epsilon}{2} \\
& \leq 2 \cdot \frac{\epsilon}{4}+\frac{\epsilon}{2}=\epsilon .
\end{aligned}
$$

Hence $\left\|\left(T_{\alpha}-T\right) x\right\| \rightarrow 0$, and the proof is complete.

With a metric and notion of convergence at hand for the strong operator topology on $X$, we turn our attention now to showing that $X$ is complete.

Proposition 4.6. The semigroup $X$ is a complete metric space with the strong operator topology.

Proof. Let $\left\{T_{n}\right\}$ be a Cauchy sequence in $X$. Then $\left\{T_{n} f\right\}$ is a Cauchy sequence in $Y$ for each $f \in Y$. By the completeness of $Y$, the sequence $\left\{T_{n} f\right\}$ converges in $Y$ for any $f \in Y$. Let 
$T: Y \rightarrow Y$ be defined by $T f=\lim _{n \rightarrow \infty} T_{n} f$. Then $T$ is linear, and since

$$
\begin{aligned}
\|T f\| & =\lim _{n \rightarrow \infty}\left\|T_{n} f\right\| \\
\leq & \lim _{n \rightarrow \infty}\left\|T_{n}\right\| \cdot\|f\| \\
& \leq \lim _{n \rightarrow \infty}\|f\|=\|f\|,
\end{aligned}
$$

we see that $T$ is continuous and $T \in X$, which completes the proof.

We now turn our attention to examples of universality on $X$ by first showing that $X$ is separable in the strong operator topology. We then study conjugation maps on $X$.

\subsection{Universality of Conjugation Maps}

Let $P_{j}: Y \rightarrow Y$ be the canonical projection onto $\operatorname{span}\left\{e_{k}: 1 \leq k \leq j\right\}$. One can easily check that if $S \in \mathcal{L}(Y, Y)$ then $P_{j} S P_{j} \rightarrow S$ strongly. Each operator $P_{j} S P_{j}$ can be represented uniquely as a $j \times j$ matrix with scalar entries with respect to the basis $\left\{e_{k}\right\}$, and so $P_{j} S P_{j}$ can be approximated arbitrarily close in norm by a $j \times j$ matrix with rational entries. Thus the countable set $\mathcal{Q}$ of all finite $j \times j$ matrices $Q_{j \times j}$ with rational entries where $j=1,2,3, \ldots$ is strongly dense in $\mathcal{L}(Y, Y)$. Since each projection $P_{j}$ has norm 1 , we see that $\mathcal{Q} \cap X$ is strongly dense in $X$. This fact yields the existence of another strongly dense subset of $X$.

Lemma 4.7. There is a strongly dense subset of $X$ consisting of operators of the form $Q+I$, where $Q \in \mathcal{Q}$.

Proof. Let $S \in X$ and $\epsilon>0$ be given, and let $d$ be a metric giving the topology of $X$. Let $Q^{\prime} \in \mathcal{Q} \cap X$ such that $d\left(Q^{\prime}, S\right)<\epsilon / 2$. Say $Q^{\prime}$ is an $m \times m$ matrix. Since $I-P_{j} \rightarrow 0$ strongly, $Q^{\prime}+I-P_{j} \rightarrow Q^{\prime}$ strongly and hence there exists some $j>m$ for which $d\left(Q^{\prime}+I-P_{j}, S\right)<\epsilon$. Now let $Q=Q^{\prime}-P_{j}$. The operator $Q$ can be represented as a $j \times j$ matrix, and $d(Q+I, S)<\epsilon$, so what remains to show is that $Q+I \in X$. 
Note that $\operatorname{ker}\left(I-P_{j}\right)=\operatorname{span}\left\{e_{1}, \ldots, e_{j}\right\}$, which contains $\operatorname{span}\left\{e_{1}, \ldots, e_{m}\right\}$. Let $f \in Y$ and write $f=g+h$ with $g \in \operatorname{span}\left\{e_{1}, \ldots, e_{m}\right\}$ and $h \in \overline{\operatorname{span}}\left\{e_{m+1}, e_{m+2}, \ldots\right\}$. Then, in the case that $Y=\ell^{p}$

$$
\begin{aligned}
\mid(Q+I) f \|_{p}^{p} & =\left\|\left(Q^{\prime}+I-P_{j}\right) f\right\|_{p}^{p} \\
& =\left\|Q^{\prime} g\right\|_{p}^{p}+\left\|\left(I-P_{j}\right) h\right\|_{p}^{p} \\
& \leq\|g\|_{p}^{p}+\|h\|_{p}^{p} \\
& =\|f\|_{p}^{p} .
\end{aligned}
$$

In the second case that $Y=c_{0}$,

$$
\begin{aligned}
\|(Q+I) f\|_{\infty} & =\left\|\left(Q^{\prime}+I-P_{j}\right) f\right\|_{\infty} \\
& \leq \max \left\{\left\|Q^{\prime} g\right\|_{\infty},\|h\|_{\infty}\right\} \\
& \leq \max \left\{\|g\|_{\infty},\|h\|_{\infty}\right\} \\
& =\|f\|_{\infty} .
\end{aligned}
$$

This shows that in both cases we have $\|Q+I\| \leq 1$, which completes the proof.

Lemma 4.7 provides us with a special dense subset of $X$ with which we can apply Corollary 2.2 to obtain a universal element of $X$.

Theorem 4.8. Let $X$ be the closed unit ball of $\mathcal{L}\left(c_{0}, c_{0}\right)$ or $\mathcal{L}\left(\ell^{p}, \ell^{p}\right)$, where $1 \leq p \leq \infty$. If $\left\{U_{n}\right\}$ is a sequence of isometric isomorphisms in $X$ such that both $\left\{U_{n}\right\}$ and $\left\{U_{n}^{-1}\right\}$ converge in the weak operator topology to 0 , then the sequence of conjugation maps $\left\{T_{n}: X \rightarrow X\right\}$ defined by $T_{n} V=U_{n}^{-1} V U_{n}$ is universal, and there is a universal element of $X$ of the form

$$
\prod_{j=1}^{\infty}\left(Q_{j}+I\right)
$$

where each $Q_{j} \in \mathcal{Q}$, and $Q_{j}+I \in X$. 
Proof. By Lemma 4.7, there is a dense subset $\mathcal{D}_{1}$ of $X$ consisting of operators of the form $Q+I$, where $Q \in \mathcal{Q}$. We take $\mathcal{D}_{2}=\mathcal{D}_{1}$ and check the three hypotheses of Corollary 2.2 .

Let $Q+I$ be a member of $\mathcal{D}_{1}$ with $Q$ a $j \times j$ matrix. Let $f \in Y$ and write $U_{n} f=g_{n}+h_{n}$, where $g_{n} \in \operatorname{span}\left\{e_{1}, \ldots, e_{j}\right\}$ and $h_{n} \in \overline{\operatorname{span}}\left\{e_{j+1}, e_{j+2}, \ldots\right\}$. We may write

$$
g_{n}=\sum_{i=1}^{j} e_{i}^{*}\left(U_{n} f\right) e_{i}
$$

where $e_{i}^{*}$ is the linear functional defined by $e_{i}^{*}\left(e_{i}\right)=1$ and $e_{i}^{*}\left(e_{k}\right)=0$ for all $k \neq i$. Then the assumption that $U_{n} \rightarrow 0$ in the weak operator topology implies that $\left\|g_{n}\right\| \rightarrow 0$. Hence $\left\|Q g_{n}\right\| \rightarrow 0$, and so

$$
\begin{aligned}
\left\|T_{n}(Q+I) f-I f\right\| & =\left\|U_{n}^{-1}(Q+I) U_{n} f-I f\right\| \\
& =\left\|U_{n}^{-1} Q U_{n} f\right\| \\
& =\left\|Q U_{n} f\right\| \\
& =\left\|Q g_{n}\right\| \rightarrow 0 .
\end{aligned}
$$

This shows that $T_{n}(Q+I) \rightarrow I$ in the strong operator topology, which is the first condition of Corollary 2.2

For the second condition, let $A_{n}=T_{n}^{-1}$, so that $A_{n} V=U_{n} V U_{n}^{-1}$. Let $f \in Y$ and write $U_{n}^{-1} f=g_{n}^{\prime}+h_{n}^{\prime}$, where $g_{n}^{\prime} \in \operatorname{span}\left\{e_{1}, \ldots, e_{j}\right\}$ and $h_{n}^{\prime} \in \overline{\operatorname{span}}\left\{e_{j+1}, e_{j+2}, \ldots\right\}$. We may write

$$
g_{n}^{\prime}=\sum_{i=1}^{j} e_{i}^{*}\left(U_{n}^{-1} f\right) e_{i}
$$

and the assumption that $U_{n}^{-1} \rightarrow 0$ in the weak operator topology implies that $\left\|g_{n}^{\prime}\right\| \rightarrow 0$. Hence $\left\|Q g_{n}^{\prime}\right\| \rightarrow 0$, and so

$$
\left\|A_{n}(Q+I) f-I f\right\|=\left\|U_{n}(Q+I) U_{n}^{-1} f-I f\right\|
$$




$$
\begin{aligned}
& =\left\|U_{n} Q U_{n}^{-1} f\right\| \\
& =\left\|Q U_{n}^{-1} f\right\| \\
& =\left\|Q g_{n}^{\prime}\right\| \rightarrow 0 .
\end{aligned}
$$

Thus the second condition of Corollary 2.2 is satisfied. Since the third condition is trivial, we have that $\left\{T_{n}\right\}$ is universal, and there is a universal element of $X$ of the form

$$
\prod_{j=1}^{\infty}\left(Q_{j}+I\right)
$$

where each $Q_{j}+I$ is in $\mathcal{D}_{1}$.

We end this chapter by mentioning that Theorem 4.8 can extend to more general operator algebras $\mathcal{L}(Y, Y)$ where the key ingredient of Lemma 4.7 still holds. For example, by passing to an equivalent norm, Theorem 4.8 holds for $\mathcal{L}(c, c)$, where $c$ is the Banach space of all convergent sequences. Let $e_{0}$ be the sequence in $c$ where each entry is the number 1 . If $f=\left(f_{1}, f_{2}, \ldots\right)$ is an element of $c$ with $\lim _{n \rightarrow \infty} f_{n}=\alpha$, write $f=g+\alpha \cdot e_{0}$, where $g \in c_{0}$, and consider the norm $\|f\|\|=\| g \|_{\infty}+|\alpha|$. A straightforward computation shows that $\|\cdot\| \cdot \|$ is indeed a norm, and that

$$
\|f\|_{\infty} \leq\|g\|_{\infty}+|\alpha| \leq 3\|f\|_{\infty},
$$

which shows that $\|\cdot\|$ is equivalent to $\|\cdot\|_{\infty}$. Equipped with the norm $\|\cdot\| \cdot \|$ on $c$, one could show the analogous results for this chapter hold on the closed unit ball of $\mathcal{L}(c, c)$. 


\section{CHAPTER 5 UNIVERSALITY ON THE CLOSED UNIT BALL OF BOUNDED MEASURABLE FUNCTIONS}

In this chapter we provide an application of the universality criterion in which the more general setting of Theorem 2.1 is required, particularly when multiplication is not continuous. Let $L^{\infty}[0,1]$ be the Banach algebra of all equivalence classes of essentially bounded Lebesgue measurable functions $f:[0,1] \rightarrow \mathbb{C}$ and let $L^{1}[0,1]$ be the Banach space of all equivalence classes of Lebesgue integrable functions $f:[0,1] \rightarrow \mathbb{C}$. Let $X=\overline{\operatorname{Ball}}\left(L^{\infty}[0,1]\right)=\left\{f \in L^{\infty}[0,1]:\|f\|_{\infty} \leq 1\right\}$ be the closed unit ball of $L^{\infty}[0,1]$.

Taking the point of view that $L^{\infty}[0,1]=\left(L^{1}[0,1]\right)^{*}$, we see that $L^{\infty}[0,1]$ carries a weak-star topology. The weak-star topology is the weakest topology such that for each $g \in L^{1}[0,1]$, the evaluation maps

$$
E_{g}: L^{\infty}[0,1] \rightarrow \mathbb{C}
$$

given by

$$
E_{g}(f)=\int f g
$$

are continuous. A net $\left\{f_{\alpha}\right\}$ in $X$ converges in the weak-star topology to $f$ if

$$
\int f_{\alpha} g \rightarrow \int f g
$$

for all $g \in L^{1}[0,1]$. By the Banach-Alaoglu theorem, $X$ is weak-star compact and weak-star complete. In addition, $X$ is also metrizable because $L^{1}[0,1]$ is separable (see [18, Theorem 2.6.23]). Furthermore, the continuous functions in $X$ form a weak-star dense subset of $X$ (see [21, Exercise 3.7]) and hence $X$ is weak-star separable by the Stone-Weierstrass Theorem.

With the above identification $L^{\infty}[0,1]=\left(L^{1}[0,1]\right)^{*}$, we see that a sequence $\left\{f_{n}\right\}$ in $X$ con- 
verges weak-star to $f$ if and only if for every $h \in L^{1}[0,1]$,

$$
\int f_{n} h \rightarrow \int f h
$$

In that case we write $f_{n} \stackrel{*}{\rightarrow} f$. With the usual multiplication of functions, $X$ is a semigroup whose identity element is the constant function 1 . However, $X$ is not a topological semigroup with the weak-star topology under the usual multiplication. To see that, let $f_{n}=e^{i 2 n \pi t}$ and $g_{n}=e^{-i 2 n \pi t}$. Then $f_{n}, g_{n} \in X$ and by the Riemann-Lebesgue lemma, $f_{n} \stackrel{*}{\rightarrow} 0$ and $g_{n} \stackrel{*}{\rightarrow} 0$, but $f_{n} g_{n}=1$. Hence the usual multiplication is not weak-star continuous. However, it is quite clear that the multiplication by a fixed $g \in X$ is weak-star continuous; that is, $L_{g}: X \rightarrow X$ defined by $L_{g}(f)=$ $g f$ is weak-star continuous.

Lemma 5.1. If $\phi:[0,1] \rightarrow[0,1]$ is a strictly increasing function, then the composition operator $T: X \rightarrow X$ defined by $T f=f \circ \phi$ is continuous.

Proof. Suppose $\left\{f_{n}\right\}$ is a sequence in $X$ and $f \in X$ with $f_{n} \stackrel{*}{\rightarrow} f$ and let $h \in L^{1}[0,1]$. Since $\phi$ is strictly increasing, $\phi^{\prime}$ exists almost everywhere. Hence, by a change of variable, we have that

$$
\begin{aligned}
\lim _{n \rightarrow \infty} \int f_{n}(\phi(x)) h(x) d x & =\lim _{n \rightarrow \infty} \int f_{n}(u) \frac{h\left(\phi^{-1}(u)\right)}{\phi^{\prime}\left(\phi^{-1}(u)\right)} d u \\
& =\int f(u) \frac{h\left(\phi^{-1}(u)\right)}{\phi^{\prime}\left(\phi^{-1}(u)\right)} d u \\
& =\int f(\phi(x)) h(x) d x,
\end{aligned}
$$

which shows that $T$ is weak-star continuous.

Obviously the composition operator $T$ in the above lemma defines a homomorphism on $X$. We study the universality properties of a sequence of such operators in Theorem 5.3, using Corollary 2.2. For that, we need to find appropriate dense subsets of $X$. 
Lemma 5.2. The set $\mathcal{D}=\left\{f \in C[0,1]:\|f\|_{\infty} \leq 1\right.$ and $\left.f(0)=1=f(1)\right\}$ is weak-star dense in $X$.

Proof. Since $C[0,1] \cap X$ is weak-star dense in $X$, it suffices to show $\mathcal{D}$ is weak-star dense in $C[0,1] \cap X$. For that, take any element $f$ in $C[0,1] \cap X$ we can modify $f$ on arbitrarily small neighborhoods of 0 and 1 , so that there is a sequence of functions $\left\{f_{n}\right\}$ in $\mathcal{D}$ such that $f_{n} \rightarrow f$ pointwise on $[0,1]$. To check that $f_{n} \stackrel{*}{\rightarrow} f$, let $h \in L^{1}[0,1]$. Then $f_{n} h \rightarrow f h$ pointwise a.e., and $\left|f_{n} h\right| \leq\left\|f_{n}\right\|_{\infty}|h|=|h|$. Thus the dominated convergence theorem implies that

$$
\int f_{n} h \rightarrow \int f h
$$

and so $f_{n} \stackrel{*}{\rightarrow} f$, completing the proof.

Lemma 5.2 provides us with a dense subset of $X$ that facilitates our application of Corollary 2.2 for the weak-star continuous compostion operators in Lemma 5.1.

Theorem 5.3. Let $X=\overline{\mathrm{Ball}}\left(L^{\infty}[0,1]\right)$ with the weak-star topology. Suppose $\left\{\phi_{n}:[0,1] \rightarrow\right.$ $[0,1]\}$ is a sequence of strictly increasing surjective functions, and that $\phi_{n} \rightarrow 1$ pointwise on $(0,1)$. Then the sequence of composition operators $\left\{T_{n}: X \rightarrow X\right\}$ given by $T_{n} f=f \circ \phi_{n}$ is universal, and there is a universal element of the form

$$
\prod_{j=1}^{\infty} f_{j}
$$

where each $f_{j} \in \mathcal{D}$ is defined in Lemma 5.2

Proof. Following the notation of Theorem 2.1. let $\mathcal{D}_{1}=\mathcal{D}_{2}=\left\{f \in C[0,1]:\|f\|_{\infty} \leq 1\right.$ and $f(0)=1=f(1)\}$, which is a weak-star dense subset of $X$ by Lemma 5.2. We check the two conditions of Theorem 2.1 are satisfied.

Let $A_{n}=T_{n}^{-1}$ and let $f, g \in \mathcal{D}_{1}$ be given. Since each $\phi_{n}$ is strictly increasing onto $[0,1]$ and $\phi_{n} \rightarrow 1$ almost everywhere on $(0,1)$, we see that $\phi_{n}^{-1} \rightarrow 0$ almost everywhere on $(0,1)$. Thus 
$g A_{n} f=g \cdot\left(f \circ \phi_{n}^{-1}\right) \rightarrow g$ almost everywhere. Since $\left|g A_{n} f\right| \leq|g|$, the dominated convergence theorem implies that $g A_{n} f \stackrel{*}{\rightarrow} g$. The exact same argument shows that $\left(T_{n} g\right)\left(T_{n} A_{n} f\right)=\left(T_{n} g\right) f \stackrel{*}{\rightarrow} f$. Hence, by Theorem 2.1, $\left\{T_{n}\right\}$ is universal and there is a universal element of $X$ of the form

$$
\prod_{j=1}^{\infty} f_{j}
$$

where each $f_{j} \in \mathcal{D}_{1}$.

Each factor $f_{j}$ of the universal element provided by the above theorem is in $C[0,1] \cap X$ and satisfies the condition $f_{j}(0)=f_{j}(1)=1$, that is independent of the given strictly increasing functions $\phi_{n}$ on $[0,1]$. Of course, the exact choice of $f_{j}$ depends on the given functions $\phi_{n}$. 


\section{CHAPTER 6 UNIVERSALITY ON THE BALL OF ALL BOUNDED AND INTEGRABLE MEASURABLE FUNCTIONS}

In this chapter, we study universality on a semigroup without an identity element. Let $X=$ $\left\{f \in L^{\infty}(\mathbb{R}):\|f\|_{\infty} \leq 1\right.$ and $\left.\|f\|_{1}<\infty\right\}$. Equip $X$ with the $L^{1}(\mathbb{R})$ norm topology and the usual multiplication of functions. It is clear that $X$ is closed under multiplication and complete, and that the multiplication map from $X \times X$ into $X$ is continuous. Thus $X$ is a separable, complete, metrizable topological semigroup without an identity, because the constant function 1 does not belong to $X$.

Along the line of the previous three chapters, we continue to consider composition operators. We first prove a lemma to determine when such operators are continuous on $L^{1}(\mathbb{R})$.

Lemma 6.1. Suppose $\phi: \mathbb{R} \rightarrow \mathbb{R}$ is a continuous, surjective, strictly increasing function. Then the composition operator $T: L^{1}[0,1] \rightarrow L^{1}[0,1]$ defined by $T f=f \circ \phi$ is continuous if and only if there exists a constant $K>0$ such that for each measurable subset $E$ of $\mathbb{R}, m\left(\phi^{-1}(E)\right) \leq$ $K(m(E))$.

Proof. If $T$ is continuous, then there is a constant $K$ such that $\|T f\|_{1} \leq K\|f\|_{1}$ for all $f \in L^{1}[0,1]$. We observe that for any measurable subset $E$ of $\mathbb{R}, \chi_{E} \circ \phi(t)=\chi_{\phi^{-1}(E)}(t)$. By setting $f=\chi_{E}$, we get that $m\left(\phi^{-1}(E)\right)=\left\|\chi_{E} \circ \phi\right\|_{1} \leq K\left\|\chi_{E}\right\|_{1}=K(m(E))$.

Conversely, suppose that there exists a constant $K>0$ such that for each measurable subset $E$ of $\mathbb{R}, m\left(\phi^{-1}(E)\right) \leq K(m(E))$. It suffices to check $\|T f\|_{1} \leq K\|f\|_{1}$ for nonnegative functions $f$. Let $f \in L^{1}[0,1]$ with $f \geq 0$ almost everywhere, and let $g_{n}$ be a sequence of nonegative simple functions converging pointwise to $f$ such that $g_{n}$ converges uniformly to $f$ on any set where $f$ is bounded and $g_{n} \leq g_{n+1}$ for all $n$. Then $\int f=\lim _{n \rightarrow \infty} \int g_{n}$. Furthermore, $g_{n} \circ \phi$ is a sequence of simple functions such that $\int f \circ \phi=\lim _{n \rightarrow \infty} \int g_{n} \circ \phi$. Since $\chi_{E} \circ \phi(t)=\chi_{\phi^{-1}(E)}(t)$, it follows that $\int g_{n} \circ \phi \leq K \int g_{n}$. Thus $\|f \circ \phi\|_{1} \leq K\|f\|_{1}$, so $T$ is continuous.

Note that any such composition operator $T$ in Lemma 6.1 maps $X$ into $X$. The next ingredient 
we need is a suitable dense subset of $X$ that facilitates the application of Theorem 2.1 on $X$, which is the content of our next lemma.

Lemma 6.2. Suppose $\left\{a_{n}:-\infty<n<\infty\right\}$ is a strictly increasing sequence of real numbers. Then the set $\mathcal{D}=\left\{f \in C(\mathbb{R}): f \in X\right.$ and $f\left(a_{n}\right)=1$ for all integers $\left.n\right\}$ is dense in $X$.

Proof. Let $g \in X$ and $\epsilon>0$ be given. There exists a function $h \in C(\mathbb{R}) \cap X$ such that $\|g-h\|_{1}<$ $\epsilon / 2$. Since $\left\{a_{n}:-\infty<n<\infty\right\}$ is strictly increasing, for each integer $n$ there exists an open interval $I_{n}$ such that $a_{n} \in I_{n}$ and $I_{n} \cap I_{j}=\emptyset$ whenever $n \neq j$. For each interval $I_{n}$, choose a subinterval $\left(c_{n}, d_{n}\right)$ containing $a_{n}$ of width no greater than $4^{-1}(1+\sqrt{2})^{-|n|-1} \epsilon$. Define a function $f: \mathbb{R} \rightarrow \mathbb{C}$ by

$$
f(x)=\left\{\begin{array}{ll}
h(x) & \text { if } x \notin \bigcup_{n=-\infty}^{\infty}\left[c_{n}, d_{n}\right] \\
\frac{1-h\left(c_{n}\right)}{a_{n}-c_{n}}\left(x-c_{n}\right)+h\left(c_{n}\right) & \text { if } x \in\left(c_{n}, a_{n}\right) \\
\frac{h\left(d_{n}\right)-1}{d_{n}-a_{n}}\left(x-d_{n}\right)+h\left(d_{n}\right) & \text { if } x \in\left[a_{n}, d_{n}\right)
\end{array} .\right.
$$

Then $f \in X, f\left(a_{n}\right)=1$ and by construction,

$$
\int_{c_{n}}^{d_{n}}|h-f|<2^{-1}(1+\sqrt{2})^{-|n|-1} \epsilon .
$$

Thus

$$
\|h-f\|_{1}<\sum_{n=-\infty}^{\infty} 2^{-1}(1+\sqrt{2})^{-|n|-1} \epsilon=\epsilon / 2,
$$

so $\|g-f\|_{1} \leq\|g-h\|_{1}+\|h-f\|_{1}<\epsilon / 2+\epsilon / 2=\epsilon$ and the proof is complete.

The above lemma leads to the following definition. A function $\psi: \mathbb{R} \rightarrow \mathbb{R}$ is called a staircase function if there exist sequences $\left\{a_{n}:-\infty<n<\infty\right\}$ and $\left\{c_{n}:-\infty<n<\infty\right\}$ of strictly increasing real numbers such that both sequences are not bounded above nor below, and

$$
\psi=\sum_{n=-\infty}^{\infty} a_{n} \chi_{\left(c_{n}, c_{n+1}\right)}
$$


Furthermore, a function $\phi: \mathbb{R} \rightarrow \mathbb{R}$ is said to be an admissible function if it is continuous, surjective, strictly increasing, and the corresponding composition operator $T: L^{1}[0,1] \rightarrow L^{1}[0,1]$ defined by $T f=f \circ \phi$ is continuous. To simplify our universality result in Theorem 6.4 below, we first prove a quick lemma.

Lemma 6.3. Suppose $\left\{\phi_{n}: \mathbb{R} \rightarrow \mathbb{R}\right\}$ is a sequence of admissible functions, and suppose there is a staircase function $\psi=\sum_{n=-\infty}^{\infty} a_{n} \chi_{\left(c_{n}, c_{n+1}\right)}$ for which $\phi_{n}$ converges pointwise to $\psi$ on $\bigcup_{n=-\infty}^{\infty}\left(c_{n}, c_{n+1}\right)$. Then $\left\{\phi_{n}^{-1}\right\}$ converges to the staircase function $\gamma=\sum_{n=-\infty}^{\infty} c_{n} \chi_{\left(a_{n-1}, a_{n}\right)}$ pointwise on $\bigcup_{n=-\infty}^{\infty}\left(a_{n-1}, a_{n}\right)$.

Proof. It suffices to show that $\phi_{n}^{-1} \rightarrow c_{2}$ pointwise on $\left(a_{1}, a_{2}\right)$. The same argument shows that $\phi_{n}^{-1} \rightarrow c_{k}$ on the interval $\left(a_{k-1}, a_{k}\right)$. Let $y$ be a point in $\left(a_{1}, a_{2}\right)$ and let $\epsilon>0$ be given. Let $\delta=\frac{1}{4} \min \left\{\epsilon,\left|a_{1}-y\right|,\left|a_{2}-y\right|\right\}$. Choose $b_{1} \in\left(c_{1}, c_{2}\right)$ with $\left|b_{1}-c_{2}\right|<\delta$ and $b_{2} \in\left(c_{2}, c_{3}\right)$ with $\left|b_{2}-c_{2}\right|<\delta$. Since $\phi_{n}\left(b_{1}\right) \rightarrow a_{1}$ and $\phi_{n}\left(b_{2}\right) \rightarrow a_{2}$, there exists a positive integer $N$ such that for all $n \geq N$,

$$
\phi_{n}\left(b_{1}\right)<\delta+a_{1}<y<a_{2}-\delta<\phi_{n}\left(b_{2}\right) .
$$

Since $\phi_{n}$ is strictly increasing, when $n \geq N, b_{1}<\phi_{n}^{-1}(y)<b_{2}$, which yields that

$$
\begin{aligned}
\left|\phi_{n}^{-1}(y)-c_{2}\right| & \leq\left|\phi_{n}^{-1}(y)-b_{2}\right|+\left|b_{2}-c_{2}\right| \\
& <\left|b_{1}-b_{2}\right|+\epsilon / 2 \\
& \leq \epsilon / 2+\epsilon / 2=\epsilon .
\end{aligned}
$$

We now apply Theorem 2.1 to prove the following.

Theorem 6.4. Let $X=\left\{f \in L^{\infty}(\mathbb{R}):\|f\|_{\infty} \leq 1\right.$ and $\left.\|f\|_{1}<\infty\right\}$. Suppose $\left\{\phi_{n}: \mathbb{R} \rightarrow \mathbb{R}\right\}$ is a sequence of admissible functions. If there exists a staircase function $\psi=\sum_{n=-\infty}^{\infty} a_{n} \chi_{\left(c_{n}, c_{n+1}\right)}$ for which $\phi_{n}$ converges pointwise to $\psi$ on $\bigcup_{n=-\infty}^{\infty}\left(c_{n}, c_{n+1}\right)$, then the set $\mathcal{D}_{1}=\{g \in C(\mathbb{R}): g \in X$ and $g\left(a_{n}\right)=1$ for all integers $\left.n\right\}$ is dense in $X$, the sequence of composition operators $\left\{T_{n}: X \rightarrow\right.$ 
$X\}$ defined by $T_{n} f=f \circ \phi_{n}$ is universal, and there is a universal element $g$ in $X$ for $\left\{T_{n}\right\}$ of the form

$$
g=\prod_{j=1}^{\infty} g_{j}
$$

where each $g_{j} \in \mathcal{D}_{1}$.

Proof. By Lemma 6.3, $\phi_{n}^{-1}$ converges to the staircase function $\gamma=\sum_{n=-\infty}^{\infty} c_{n} \chi_{\left(a_{n-1}, a_{n}\right)}$ pointwise on $\bigcup_{n=-\infty}^{\infty}\left(a_{n-1}, a_{n}\right)$. Let

$$
\mathcal{D}_{2}=\left\{f \in C(\mathbb{R}): f \in X \text { and } f\left(c_{n}\right)=1 \text { for all integers } n\right\} .
$$

By Lemma 6.2, the sets $\mathcal{D}_{1}$ and $\mathcal{D}_{2}$ are dense in $X$.

We now check the two conditions of Theorem 2.1 for any given functions $g \in \mathcal{D}_{1}$ and $f \in$ $\mathcal{D}_{2}$. Since $\phi_{n}^{-1} \rightarrow \gamma$ pointwise almost everywhere, $f\left(\phi_{n}^{-1}(t)\right) \rightarrow f(\gamma(t))=1$ pointwise almost everywhere. Furthermore,

$$
\begin{aligned}
\left|g \cdot\left(f \circ \phi_{n}^{-1}\right)\right| & \leq\left\|f \circ \phi_{n}^{-1}\right\|_{\infty}|g| \\
& \leq\|f\|_{\infty}|g|,
\end{aligned}
$$

hence the dominated convergence theorem implies that $\left\|g T_{n}^{-1} f-g\right\|_{1} \rightarrow 0$.

For the second condition, we note that $\phi_{n} \rightarrow \psi$ almost everywhere, and so $g\left(\phi_{n}(t)\right) \rightarrow \psi(t)=$ 1 almost everywhere. Since

$$
\left|\left(T_{n} g\right)\left(T_{n} A_{n} f\right)\right| \leq\|g\|_{\infty}|f|,
$$

by the dominated convergence theorem, we see that

$$
\left\|\left(T_{n} g\right)\left(T_{n} A_{n} f\right)-f\right\|_{1} \rightarrow 0 .
$$


Thus by Theorem 2.1, we see that $\left\{T_{n}\right\}$ is universal and there is a universal element of the form

$$
g=\prod_{j=1}^{\infty} g_{j}
$$

where each $g_{j} \in \mathcal{D}_{1}$.

To conclude our discussion, we observe that if the semigroup $X$ in Theorem 2.1 is not assumed to be complete, but merely a dense semigroup $X$ in a complete metric space $Y$, then the proof of the theorem yields a universal element $x$ for $Y$. Apply this observation to the semigroup $X=$ $C(\mathbb{R}) \cap L^{\infty}(\mathbb{R})$, with the usual multiplication of functions, as a dense subset of the complete metric space $Y=L^{1}(\mathbb{R})$. Then, for a sequence $\left\{\phi_{n}\right\}$ of admissible functions satisfying the hypotheses of Theorem 6.4 and corresponding composition operators $T_{n}: f \mapsto f \circ \phi_{n}$, there is a universal element $g$ in $Y$ for $\left\{T_{n}: Y \rightarrow Y\right\}$ which is the infinite product of continuous functions. 


\section{CHAPTER 7 FUTURE WORK}

We mention here a few questions regarding specific applications of Corollary 2.2 related to the results mentioned above. The first is about special kinds of Blaschke products which are of interest to many function theorists.

The sequence $\left\{z_{n}\right\}$ in $\mathbb{D}$ is an interpolating sequence in $H^{\infty}(\mathbb{D})$ if every interpolation problem

$$
f\left(z_{n}\right)=a_{n}
$$

for $\left\{a_{n}\right\} \in \ell^{\infty}$ has a solution $f \in H^{\infty}(\mathbb{D})$. An interpolating Blaschke product is a Blaschke product whose zeros form an interpolating sequence. To see applications and the importance of interpolating Blaschke products, we refer the reader to the last three chapter's of Garnett's book [9]. While we have shown the existence of a universal Blaschke product, we are interested to know whether or not such a Blaschke product could be interpolating.

Question 7.1. Is there a universal interpolating Blaschke product? That is, in the setting of Theorem 3.10 can the Blaschke product B be an interpolating Blaschke product?

Next, we note that the Universality Criterion is only a sufficient condition for universality. It was a long-standing open problem to exhibit an example of a universal sequence of operators which fail the (weak form of the) criterion. This was done in 2009 by De La Rosa and Read [8], but the example is very technical. It would be nice to have a more natural example.

Question 7.2. Can we simplify the result of De La Rosa and Read?

One way to tackle the problem is to find a Fréchet algebra which satisfies Corollary 2.2 (for multiplication) but fails to satisfy the criterion for addition.

Lastly, we would like to know more about the universal operator for conjugation constructed 
in Theorem 4.8. Recall that the universal operator constructed is of the form

$$
\prod_{j=1}^{\infty}\left(Q_{j}+I\right)
$$

where each $Q_{j}$ is a finite square matrix and $\left\|Q_{j}+I\right\| \leq 1$.

Question 7.3. Is the universal operator given by

$$
\prod_{j=1}^{\infty}\left(Q_{j}+I\right)
$$

in Theorem 4.8 compact? Is it a compact perturbation of the identity operator? 


\section{BIBLIOGRAPHY}

[1] A. B. Aleksandrov. Function theory in the ball. In Current problems in mathematics. Fundamental directions, Vol. 8, Itogi Nauki i Tekhniki, pages 115-190, 274. Akad. Nauk SSSR, Vsesoyuz. Inst. Nauchn. i Tekhn. Inform., Moscow, 1985.

[2] Frédéric Bayart. Universal inner functions on the ball. Canad. Math. Bull., 51(4):481-486, 2008.

[3] Frédéric Bayart and Pamela Gorkin. How to get universal inner functions. Math. Ann., 337(4):875-886, 2007.

[4] Frédéric Bayart, Pamela Gorkin, Sophie Grivaux, and Raymond Mortini. Bounded universal functions for sequences of holomorphic self-maps of the disk. Ark. Mat., 47(2):205-229, 2009.

[5] George D. Birkhoff. Démonstration d?un théoreme elémentaire sur les fonctions entières. Acad. Sci. Paris, 189:473-475, 1929.

[6] Kit C. Chan. Universal meromorphic functions. Complex Variables Theory Appl., 46(4):307314, 2001.

[7] P. S. Chee. Universal functions in several complex variables. J. Austral. Math. Soc. Ser. A, 28(2):189-196, 1979.

[8] Manuel de la Rosa and Charles Read. A hypercyclic operator whose direct sum $T \oplus T$ is not hypercyclic. J. Operator Theory, 61(2):369-380, 2009.

[9] John B. Garnett. Bounded analytic functions, volume 96 of Pure and Applied Mathematics. Academic Press, Inc. [Harcourt Brace Jovanovich, Publishers], New York-London, 1981.

[10] P. M. Gauthier and J. Xiao. The existence of universal inner functions on the unit ball of $\mathbb{C}^{n}$. Canad. Math. Bull., 48(3):409-413, 2005. 
[11] Robert M. Gethner and Joel H. Shapiro. Universal vectors for operators on spaces of holomorphic functions. Proc. Amer. Math. Soc., 100(2):281-288, 1987.

[12] Pamela Gorkin and Raymond Mortini. Universal Blaschke products. Math. Proc. Cambridge Philos. Soc., 136(1):175-184, 2004.

[13] Pamela Gorkin and Raymond Mortini. Universal singular inner functions. Canad. Math. Bull., 47(1):17-21, 2004.

[14] Karl-G. Grosse-Erdmann and Alfredo Peris Manguillot. Linear chaos. Universitext. Springer, London, 2011.

[15] Maurice Heins. A universal Blaschke product. Arch. Math. (Basel), 6:41-44, 1954.

[16] Carol Kitai. INVARIANT CLOSED SETS FOR LINEAR OPERATORS. ProQuest LLC, Ann Arbor, MI, 1982. Thesis (Ph.D.)-University of Toronto (Canada).

[17] G. R. MacLane. Sequences of derivatives and normal families. J. Analyse Math., 2:72-87, 1952.

[18] Robert E. Megginson. An introduction to Banach space theory, volume 183 of Graduate Texts in Mathematics. Springer-Verlag, New York, 1998.

[19] James R. Munkres. Topology: a first course. Prentice-Hall, Inc., Englewood Cliffs, N.J., 1975.

[20] S. Rolewicz. On orbits of elements. Studia Math., 32:17-22, 1969.

[21] Walter Rudin. Functional analysis. McGraw-Hill Book Co., New York-DüsseldorfJohannesburg, 1973. McGraw-Hill Series in Higher Mathematics.

[22] W. Seidel and J. L. Walsh. On approximation by euclidean and non-euclidean translations of an analytic function. Bull. Amer. Math. Soc., 47:916-920, 1941. 
[23] T. K. Subrahmonian Moothathu. Weak mixing and mixing of a single transformation of a topological (semi)group. Aequationes Math., 78(1-2):147-155, 2009. 